\title{
Neurotrophic tyrosine kinase receptor 1 is a direct transcriptional and epigenetic target of IL-13 involved in allergic inflammation
}

\author{
M Rochman ${ }^{1}$, AV Kartashov ${ }^{1}, J_{M}$ Caldwell ${ }^{1}$, MH Collins $^{2}$, EM Stucke ${ }^{1}, \mathrm{~K} \mathrm{KC}^{1}$, JD Sherrill ${ }^{1}$, J Herren ${ }^{1}$, \\ A Barski ${ }^{1}$ and ME Rothenberg ${ }^{1}$
}

Although interleukin (IL)-13 and neurotrophins are functionally important for the pathogenesis of immune responses, the interaction of these pathways has not been explored. Herein, by interrogating IL-13-induced responses in human epithelial cells we show that neurotrophic tyrosine kinase receptor, type 1 (NTRK1), a cognate, high-affinity receptor for nerve growth factor (NGF), is an early transcriptional IL-13 target. Induction of NTRK1 was accompanied by accumulation of activating epigenetic marks in the promoter; transcriptional and epigenetic changes were signal transducer and activator of transcription 6 dependent. Using eosinophilic esophagitis as a model for human allergic inflammation, we found that NTRK1 was increased in inflamed tissue and dynamically expressed as a function of disease activity and that the downstream mediator of NTRK1 signaling early growth response 1 protein was elevated in allergic inflammatory tissue compared with control tissue. Unlike NTRK1, its ligand NGF was constitutively expressed in control and disease states, indicating that IL-13-stimulated NTRK1 induction is a limiting factor in pathway activation. In epithelial cells, NGF and IL-13 synergistically induced several target genes, including chemokine (C-C motif) ligand 26 (eotaxin-3). In summary, we have demonstrated that IL-13 confers epithelial cell responsiveness to NGF by regulating NTRK1 levels by a transcriptional and epigenetic mechanism and that this process likely contributes to allergic inflammation.

\section{INTRODUCTION}

Interleukin 13 (IL-13)-mediated allergic inflammation is a hallmark of a number of diseases, including asthma, atopic dermatitis, and eosinophilic esophagitis (EoE) ${ }^{1-3}$ IL-13 induces robust, cell-specific changes in gene expression, and a majority of IL-13-mediated transcriptional and pathological changes are signal transducer and activator of transcription 6 (STAT6) dependent. ${ }^{4-6}$ For example, in a murine model of EoE induced by IL-13 delivery into the lungs, eosinophilic infiltration and epithelial hyperplasia in the esophagus occur in a STAT6dependent manner. ${ }^{7}$ Likewise, in human intestinal, airway, and esophageal epithelial cells, induction of the eosinophil-specific chemokine (C-C Motif) ligand 26 (CCL26 (eotaxin-3)) by IL-13 requires STAT6 expression. ${ }^{8-10}$ To date, nearly all targets of IL-13 have been signaling molecules and/or soluble mediators of inflammation. Herein, we focus on a novel induction pathway in which IL-13 confers epithelial cell responsiveness to nerve growth factor (NGF) by inducing the NGF cognate, high-affinity receptor neurotrophic tyrosine kinase receptor, type 1 (NTRK1).

NGF was originally described as a critical factor for the survival and maintenance of sympathetic and sensory neurons, ${ }^{11}$ yet NGF is also considered a biomarker of asthmatic inflammation, with increased levels correlating with the severity of the disease. ${ }^{12}$ Accordingly, NTRK1 is expressed on various structural and hematopoietic cells, including basophils and eosinophils. ${ }^{13,14}$ Moreover, early growth response protein 1 (EGR1), a central transcriptional target of NGF, ${ }^{15}$ has a key role in IL-13-induced inflammation, fibrosis, alveolar remodeling, and cytokine response; ${ }^{16}$ however, a connection between

$\overline{1}$ Divisions of Allergy and Immunology, Cincinnati Children's Hospital Medical Center, University of Cincinnati College of Medicine, Cincinnati, Ohio, USA and ${ }^{2}$ Divisions of Pathology and Laboratory Medicine, Cincinnati Children's Hospital Medical Center, University of Cincinnati College of Medicine, Cincinnati, Ohio, USA. Correspondence: ME Rothenberg (Marc.Rothenberg@cchmc.org)

Received 22 June 2014; accepted 9 October 2014; published online 12 November 2014. doi:10.1038/mi.2014.109 
NTRK1 and these EGR1-associated inflammatory and/or remodeling pathways has not been established.

We herein employed RNA-sequencing analysis of the IL-13mediated transcriptional response in the human esophageal epithelial cell line TE-7. We identified a set of 24 early IL-13 target genes, which included NTRK1. In response to IL-13, NTRK1 was dramatically induced in epithelial cells, and the NTRK1 promoter showed rapid accumulation of multiple activating epigenetic marks; both transcriptional and epigenetic changes occurred in a STAT6-dependent manner. Notably, NTRK1 was the only receptor tyrosine kinase (RTK) with these characteristics. Functional analysis showed that IL-13-induced NTRK1 responded to NGF by activating EGR1 signaling and synergistically inducing a number of IL-13 target genes, including CCL26, serpin peptidase inhibitor, clade B, member 4 (SERPINB4), and KIT ligand (KITLG). EGR1 induction was significantly diminished by gene silencing or by pharmacological inhibition of NTRK1. Translational studies showed elevated expression of NTRK1 in allergic tissue from patients with EoE. In summary, we have identified NTRK1 as a direct transcriptional and epigenetic target of IL-13 with a contributory role in allergic inflammation.

\section{RESULTS}

\section{Transcriptional signature of IL-13 response in TE-7 esophageal epithelial cells}

To gain insight into the transcriptional signature of IL-13mediated allergic inflammation, we analyzed the kinetics of the transcriptional response to IL-13 in the human esophageal epithelial cell line TE-7. ${ }^{17}$ Cells were stimulated with IL-13 for 2,6 , and $24 \mathrm{~h}$ and subjected to RNA-sequencing analysis. By applying differential expression analysis for sequence count data (DESeq) ${ }^{18}$ we identified 767 unique genes significantly affected by IL-13 during the course of stimulation $(P<0.05$, Figure 1a and see Supplementary Table S1 online); 24, 328, and 573 genes were affected after 2, 6, and $24 \mathrm{~h}$ of stimulation, respectively. Comparing the transcriptional response in TE-7 cells with the transcriptome of diseased tissue (esophageal biopsies from patients with active EoE) and of IL-13-induced primary esophageal epithelial cells revealed a remarkable similarity in the regulation of overlapping genes, including induction of the EoE hallmark genes CCL26 and cadherin 26 $(\mathrm{CDH} 26)^{10,19}$ (Figure $\mathbf{1 b}$ and see Supplementary Figure S1). Kinetic analysis of the IL-13-mediated transcriptome of TE-7 cells revealed dynamic changes in gene expression, with early response genes mostly affected after $2 \mathrm{~h}$ of stimulation and late response genes significantly changed only after $24 \mathrm{~h}$ of IL- 13 stimulation.

Of the 24 early target genes that responded to IL-13 within $2 \mathrm{~h}$ of stimulation, 20 genes were significantly altered at 6 and $24 \mathrm{~h}$. Of these 20 genes, only inhibitor of DNA binding 3 (ID3) was decreased after $24 \mathrm{~h}$, whereas other genes were significantly upregulated at all the assessed time points (Figure 1c). In agreement with previous findings, CCL26 was one of the early transcriptional targets of IL-13. ${ }^{10}$ Among early target genes, we found known inhibitors of cytokine signaling, suppressor of cytokine signaling 1 (SOCS1), and cytokine inducible SH2containing protein $(\mathrm{CISH})$, which can provide a negative feedback loop for cytokine signaling. ${ }^{20}$ We also detected genes such as hyaluronan synthase 3 (HAS3), heparan sulfate (glucosamine) 3-O-sulfotransferase 1 (HS3ST1), and glucosaminyl ( $N$-acetyl) transferase 3, mucin type (GCNT3), which are involved in the synthesis of unbranched glycosaminoglycan hyaluronan, heparan sulfate, and mucin, major constituents of the epithelial extracellular environment. ${ }^{21-23}$ These genes have been previously identified in EoE biopsies and in primary human esophageal epithelial cells induced with IL-13. ${ }^{10,19}$ Notably, among early responsive genes, there was a member of the AT-rich interaction domain family of DNA-binding proteins, AT-rich interactive domain $5 \mathrm{~B}$ (ARID5B), which functions as a cofactor in a histone demethylation process, ${ }^{24}$ and mitogen-activated protein kinase kinase kinase 14 (MAP3K14), a nuclear factor kappa-B (NF-кB)-inducing kinase that participates in regulating tumor necrosis factor and IL-1 receptor signaling, ${ }^{25}$ suggesting that initial stimulation by IL-13 can affect subsequent stimuli by modulating chromatin structure and signaling events. A member of the epithelial-specific ETS transcription factors ETS homologous factor $(E H F),{ }^{26}$ a RUNX family protein runt-related transcription factor 2 (RUNX2), and the zinc finger protein $\mathrm{B}$-cell CLL/lymphoma 11B (BCL11B) were also identified as early targets of IL-13 in TE-7 cells. These transcription factors have been previously implicated in regulating epithelial cell proliferation and differentiation. ${ }^{27-29} I L-31$, which is normally produced by activated $\mathrm{T}$ cells, ${ }^{30}$ was highly induced in TE-7 epithelial cells. Interestingly, epithelial cells have been shown to respond synergistically to IL-13 and IL-31 by secreting inflammatory cytokines, such as epidermal growth factor, vascular endothelial growth factor, and CCL $2,{ }^{31}$ suggesting a positive feedback loop in inducing allergic inflammation.

We identified NTRK1 as one of most highly IL-13-induced genes in TE-7 cells. NTRK1 is highly expressed in the nervous system and has a role in the survival of neurons, as well as in sensitivity to pain. ${ }^{32}$ A growing body of evidence links the NGF/ NTRK1 pathway to the pathogenesis of allergic inflammation, such as asthma and allergic rhinitis, ${ }^{33-35}$ yet a direct link between IL-13- and NGF-mediated pathways has not been shown.

NTRK1 is a unique RTK transcriptionally induced by IL-13 In light of the emerging role of NGF/NTRK1 signaling in the pathogenesis of allergic inflammation, we were intrigued by the robust induction of NTRK1 by IL-13 in TE-7 cells. We hypothesized that the NGF/NTRK1 and IL-13/STAT6 pathways cooperate in propagating allergic inflammation. We therefore focused on exploring the mechanism of IL-13mediated NTRK1 induction in epithelial cells and the functional outcome of this induction. First, we validated our RNA-sequencing finding by testing the kinetics of NTRK1 expression in TE-7 cells by real-time PCR (RT-PCR). In agreement with RNA-sequencing data, this analysis revealed that the NTRK1 transcript was detectable as early as $2 \mathrm{~h}$ after 


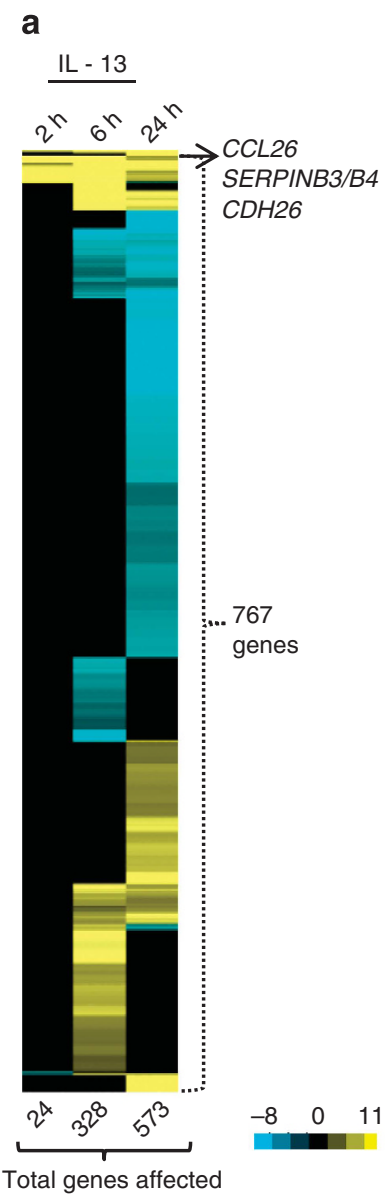

b
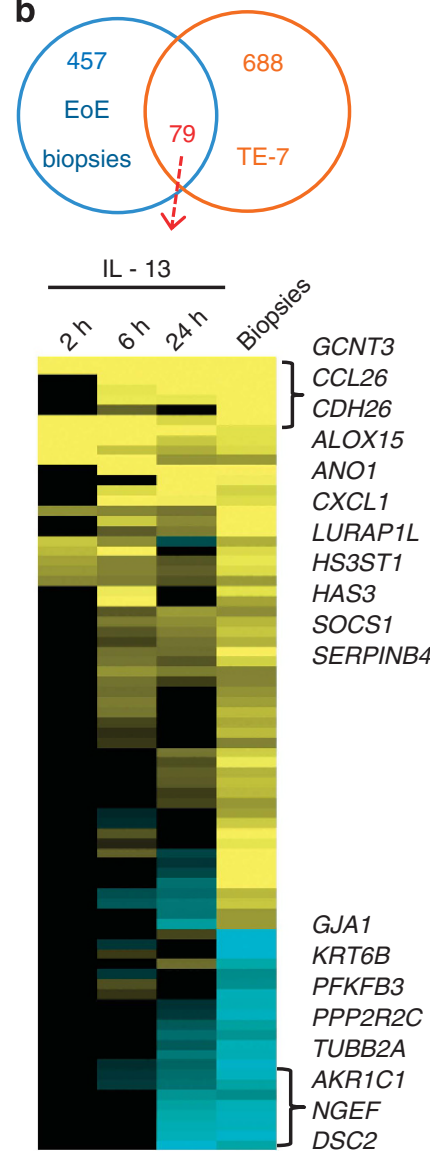

c
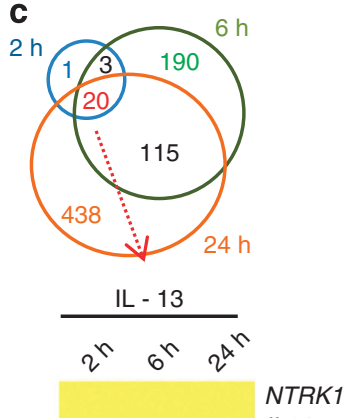

IL31

CCL26

GCNT3

HS3ST1

SERPINB3

LURAP1L

LIFR

$\mathrm{CISH}$

HAS3

SOCS1

SLC9A3

RUNX2

MFHAS1

KITLG

MAP3K 14

EHF

BCL11B

$A R I D 5 B$ ID3

Figure 1 Kinetics of interleukin (IL)-13 response in TE-7 epithelial cells. (a) Heat map represents hierarchical clustering of the fold change difference of 767 genes significantly altered by IL-13 treatment in the TE-7 cell line compared with untreated control (DESeq $P<0.05$ ). (b) Venn diagram shows the overlap of genes significantly affected by IL-13 in TE-7 cells at all the assessed time points and genes differentially expressed in human esophageal biopsies of patients with active eosinophilic esophagitis (EoE; $P<0.05)$. The heat map represents the log(2) fold change of 79 commonly affected genes compared with untreated TE-7 cells or control biopsies. The most highly upregulated and downregulated genes are indicated. (c) Venn diagram represents the overlap of genes significantly affected by IL-13 after 2,6 , or $24 \mathrm{~h}$ of stimulation. The heat map shows the kinetics of the transcriptional response for 20 genes affected at all the IL-13 treatment time points. Note the increased expression of neurotrophic tyrosine kinase receptor, type 1 (NTRK1) throughout IL-13 stimulation. For all heat maps, clustering was performed using Euclidean distance and average linkage parameters. Yellow and blue colors correspond to increased or decreased expression, respectively, and black color indicates non-significant change compared with controls.

stimulation and progressively increased at the assessed time points over a 24 -h period mirroring induction of CCL26 (Figure 2a). We further assessed the expression of NTRK1 in the esophageal epithelial cell line EPC2 grown at air-liquid interface, primary epithelial cells obtained from esophageal biopsies, and human bronchial epithelial cells after stimulation with IL-13. The results demonstrated that NTRK1 was highly induced by IL-13 in epithelial cells of different origin; the kinetics of NTRK1 induction paralleled those of CCL26 (Figure 2b-d). Finally, we tested whether induction of NTRK1 was dependent on IL-13 signaling. By stably integrating shRNA against STAT6 in TE-7 cells, we decreased the levels of STAT 6 mRNA and protein by $20-30 \%$ of that of control cells (see Supplementary Figure S2). By stimulating these cells with IL-13, we found that transcriptional induction of both CCL26 and NTRK1 was significantly decreased, indicating STAT6 dependency (Figure 2e).
As approximately 90 RTKs have been identified in the human genome, ${ }^{36}$ we investigated how many of these molecules besides NTRK1 were altered in the course of IL-13 induction. From RNA-sequencing data, we identified 29 RTKs that were expressed in either untreated or IL-13-stimulated TE-7 cells (reads per kilobase per million mapped reads $(\mathrm{RPKM})>1$ ) and analyzed the effect of IL-13 on their transcription. We found that transcription of six members of the RTK family was significantly affected by IL-13 in at least one time point of stimulation $(P<0.05)$; however, NTRK1 was unique in that it was the only RTK strongly and consistently upregulated throughout IL-13 stimulation (Figure 3a,b).

\section{IL-13 epigenetically regulates NTRK1 in a STAT6-dependent manner}

We analyzed the levels of the activating epigenetic marks $\mathrm{H} 3 \mathrm{~K} 9 \mathrm{Ac}, \mathrm{H} 3 \mathrm{~K} 27 \mathrm{Ac}$, and $\mathrm{H} 3 \mathrm{~K} 4 \mathrm{me}$, which reside on actively 

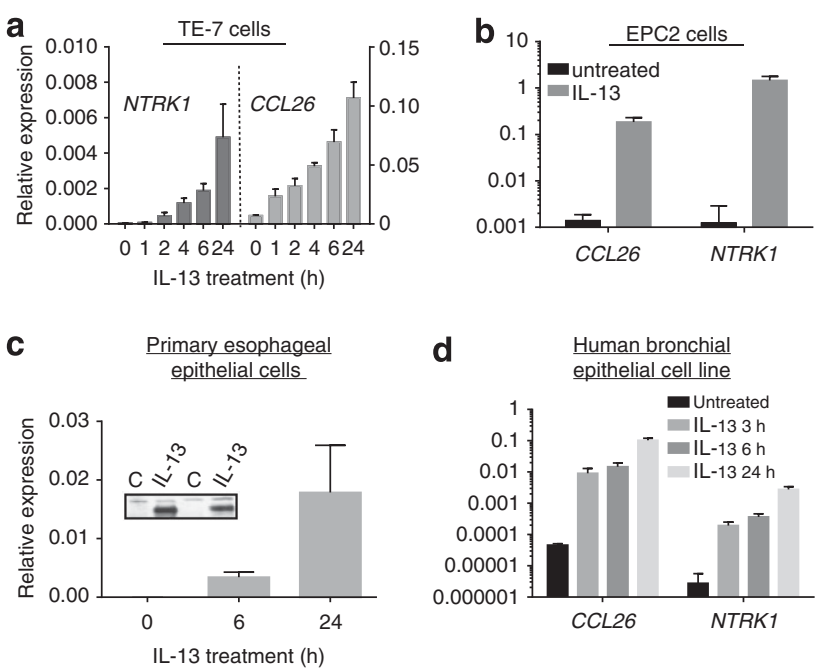

e
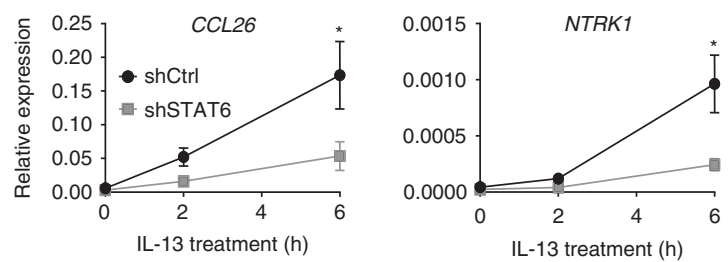

Figure 2 Interleukin (IL)-13-stimulated neurotrophic tyrosine kinase receptor, type 1 (NTRK1) induction in epithelial cells and effect of signal transducer and activator of transcription 6 (STAT6) gene silencing. (a-d) Quantitative real-time PCR (RT-PCR) analysis of NTRK1 and chemokine (C-C motif) ligand 26 (CCL26) transcription in TE-7 cells, EPC2 epithelial cell air-liquid interface culture, primary esophageal epithelial cells, and human bronchial epithelial cells. Cells were treated with IL-13 at $100 \mathrm{ng} \mathrm{ml}^{-1}$ for the indicated periods of time or for 6 days for EPC2 cells. The inset in c shows western blotting for NTRK1 in two independent cultures of primary esophageal epithelial cells stimulated with IL-13. (e) Effect of STAT6 gene silencing by shRNA on NTRK1 and CCL26 induction in TE-7 cells was quantified by RT-PCR. TE-7 cells were stimulated with IL-13 (1 ng ml $\left.{ }^{-1}\right)$ for the indicated periods of time. C, control; shCtrl, control shRNA; shSTAT6, shRNA against STAT6. Data for 3-4 independent experiments are presented as mean values for gene expression normalized to the level of $G A P D H$ with s.e. measurements. ${ }^{\star} P<0.05$

transcribed genes ${ }^{37}$ in the promoter of NTRK1. Quantitative kinetic analysis revealed the presence of activating chromatin marks prior to IL-13 stimulation and the progressive increase in the levels of $\mathrm{H} 3$ acetylation and methylation with IL-13 stimulation, starting from the 2 -h time point (Figure 4a, NTRK1). Similarly, the promoter of CCL26 (positive control) was marked by histone acetylation, especially $\mathrm{H} 3 \mathrm{~K} 27 \mathrm{Ac}$, prior to IL-13 stimulation, and the level of acetylation was significantly increased after IL-13 stimulation; the level of H3K4me3 was not significantly affected by IL-13 (Figure 4a, CCL26). As a negative control, activating epigenetic marks were absent in the promoter of the myogenic differentiation 1 (MYOD) gene, which was neither expressed nor induced in TE7 cells (Figure 4a, MYOD). We next examined the requirement for STAT6 in epigenetic regulation of NTRK1. We utilized TE-7 STAT6 knockdown cells to assess the level of acetylation and methylation chromatin marks in the NTRK1 promoter a
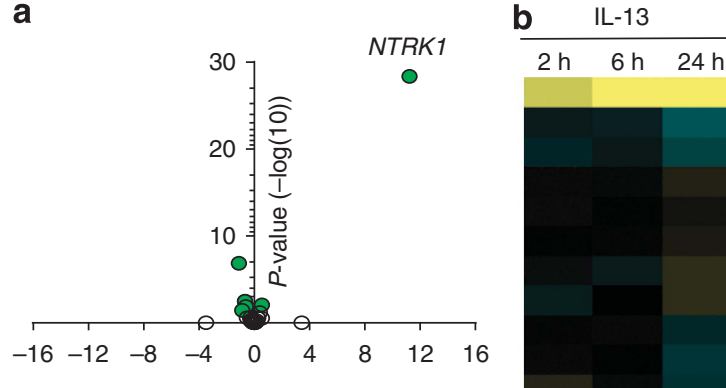

Expression fold change $(\log (2))$

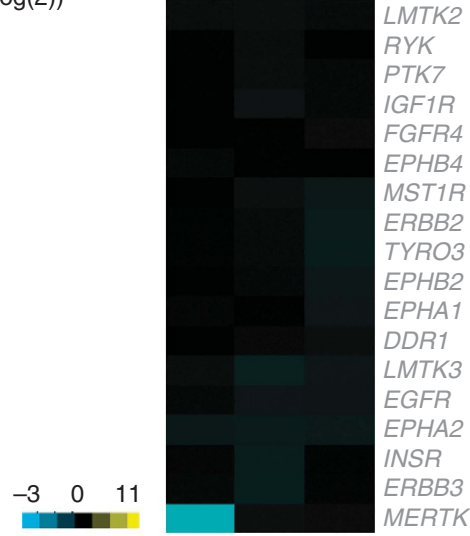

Figure 3 Effect of interleukin (IL)-13 on transcription of receptor tyrosine kinases (RTKs). (a) Volcano plot represents the expression of 29 RTKs with detectable expression in TE-7 cells (reads per kilobase per million mapped reads $>1$ ) after IL-13 stimulation for $24 \mathrm{~h}$. The $x$ axis shows $\log (2)$ fold change of expression, and the $y$ axis shows negative log (10) $P$-value for each gene. Green dots represent RTKs displaying significant change in expression in response to IL-13 in at least one time point $(\mathrm{DESeq}<0.05)$. (b) Heat map shows the kinetics of the transcriptional response for 29 RTKs after IL-13 stimulation. RTKs displayed as green dots in a are indicated in bold. Yellow and blue colors correspond to increased or decreased expression, respectively. The list of RTKs was compiled from Robinson et $\mathrm{al}^{73}$ and filtered by the level of expression.

following IL-13 stimulation. We found that the IL-13-mediated increase in activating chromatin modifications was dependent on STAT6 (Figure $\mathbf{4 b}$ ). Collectively, these data identified NTRK1 as a direct early transcriptional and epigenetic target of IL-13 in human epithelial cells.

\section{IL-13-induced NTRK1 confers epithelial cell responsiveness to NGF}

NTRK1 belongs to the family of MAPK receptors, which undergo dimerization and phosphorylation on tyrosine residues in response to NGF stimulation. Subsequently, downstream signaling events are induced, including stimulation of the RAS/ RAF pathway, PLC $\gamma$ recruitment, and phosphatidylinositol $3^{\prime}$-kinase activation. ${ }^{34}$ To assess the functionality of IL-13induced NTRK1 in epithelial cells, we tested the ability of NGF to induce signal transduction in primary epithelial and TE-7 cells pretreated with IL-13. Western blotting analysis showed that NTRK1 was not detected in resting cells but was induced after IL-13 stimulation in primary esophageal epithelial cells and TE-7 cells (Figure 5a,b, NTRK1 panel). Subsequent 

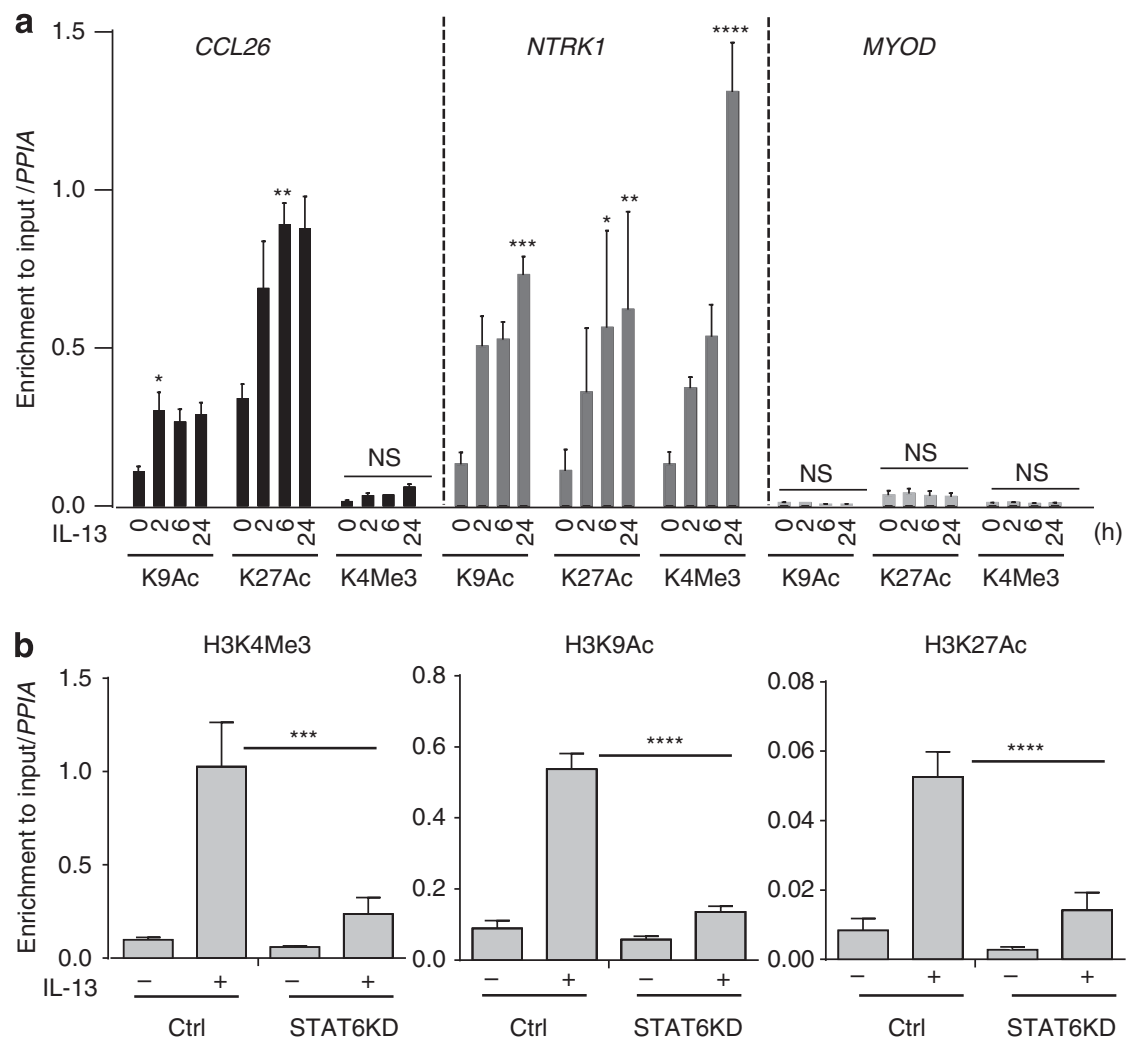

Figure 4 Effect of interleukin (IL)-13 stimulation and STAT6 silencing on epigenetic status of neurotrophic tyrosine kinase receptor, type 1 (NTRK1). (a) Levels of H3K9Ac, H3K27Ac, and H3K4me3 in the promoters of chemokine (C-C motif) ligand 26 (CCL26), NTRK1, and MYOD after IL-13 stimulation were quantified by chromatin immunoprecipitation-real-time PCR (RT-PCR). Data from three independent experiments calculated as the percentage of signal in input DNA normalized to the level of signal in PPIA gene are presented as mean values with s.e. measurements. (b) Levels of histone modification in the NTRK1 promoter following induction with IL-13 for $24 \mathrm{~h}$ were quantified by RT-PCR in control (Ctrl) and TE-7 cells where the signal transducer and activator of transcription 6 (STAT6) gene was silenced by shRNA (STAT6KD). Combined data for two independent experiments are shown. ${ }^{*} P<0.05,{ }^{* *} P<0.01,{ }^{* * *} P<0.001,{ }^{* * * *} P<0.0001$, NS, not significant.

a

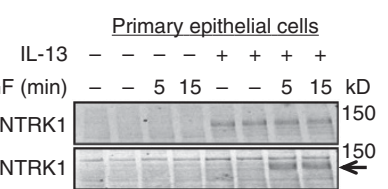

b

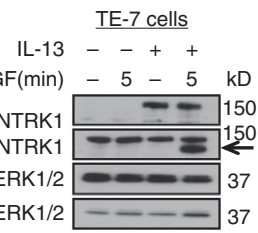

C TE-7 cells $\mathrm{NGF}(\min )-30-51530 \mathrm{kD}$

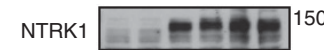
PNTRK1
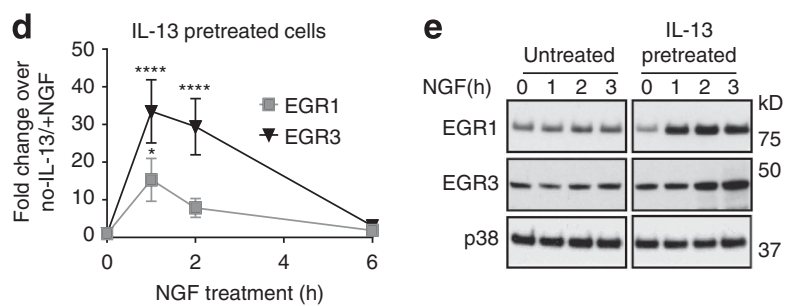

Figure 5 Neurotrophic tyrosine kinase receptor, type 1 (NTRK1) confers epithelial cell responsiveness to nerve growth factor (NGF). (a) Western blotting analysis of primary esophageal epithelial cells pretreated with interleukin (IL)-13 for $24 \mathrm{~h}$ and then treated with recombinant human NGF for 0,5 , or 15 min. pNTRK1 indicates phosphorylated protein (arrow). (b) Western blotting analysis of TE-7 cells pretreated with IL-13 for $24 \mathrm{~h}$ and then treated with NGF for 0 or $5 \mathrm{~min}$. pNTRK1 and pERK1/2 indicate phosphorylated proteins, arrow points at pNTRK1. (c) Kinetics of NTRK1 phosphorylation were assessed by western blotting. Cells were pretreated with IL-13 for $24 \mathrm{~h}$ and then treated with NGF for 0,5 , 15 , or 30 min. For a-c, phosphorylation was assessed at tyrosine residues Tyr674/675 in the catalytic domain of NTRK1. (d) Kinetic analysis of EGR1 and EGR3 mRNA in TE-7 cells pretreated with IL-13 for $24 \mathrm{~h}$ followed by treatment with $\mathrm{NGF}$ for $0,1,2$, or $6 \mathrm{~h}$ was performed by real-time PCR. Fold change indicates increase over untreated (no IL-13) cells stimulated with NGF ( + NGF). NGF was used at the concentration of $100 \mathrm{ng} \mathrm{ml}^{-1}$. Data for three independent experiments are presented as mean value with s.e. measurements; ${ }^{* * *} P<0.0001,{ }^{*} P<0.05$. (e) EGR1 and EGR3 protein levels in TE-7 cells pretreated with IL-13 for $24 \mathrm{~h}$ followed by treatment with NGF were analyzed by western blotting; p38 serves as a loading control. 
stimulation with NGF caused rapid phosphorylation of tyrosine residues Tyr674/675 in the catalytic domain of NTRK1 (Figure 5a,b, pNTRK1 panel), as well as increased phosphorylation of extracellular signal-regulated kinase 1/2 (ERK1/2; Figure 5b, pERK1/2 panel) in IL-13-treated cells. In agreement with previous reports, NTRK1 showed rapid kinetics of Tyr674/675 phosphorylation with peak expression at $5 \mathrm{~min}$ and a progressive decrease over $30 \mathrm{~min}$ after NGF stimulation ${ }^{38}$ (Figure 5c). To demonstrate that NGF stimulation caused a transcriptional response, we measured the levels of EGR1 and EGR3 mRNA, which are known to be transcriptional targets of NGF in PC12 pheochromocytoma cells. ${ }^{15,39}$ In IL-13-pretreated cells, but not in untreated cells, we observed a rapid and transient increase in EGR1 and EGR3 transcripts, which peaked at $1 \mathrm{~h}$ after NGF addition (Figure 5d). Accordingly, increased EGR1 and EGR3 protein levels were detected after NGF stimulation of IL-13-pretreated cells (Figure 5e). Taken together, these data show that IL-13 induces functional NTRK1 and thereby confers NGF sensitivity to human epithelial cells.

\section{NTRK1 and EGR1, but not NGF, are increased in esophageal biopsies from EoE patients}

In vitro experiments with epithelial cells prompted us to investigate whether NTRK1 is increased in epithelial cells in vivo during allergic responses. First, we assessed the expression of NTRK1 mRNA in esophageal biopsies of control and EoE patients. RT-PCR analysis showed a highly significant increase of NTRK1 expression in the biopsies of patients with active EoE compared with unaffected control individuals (Figure 6a, $P<0.001$ ). In addition, NTRK1 was dynamically expressed as a function of disease activity, as the esophagus of patients who responded to swallowed steroid treatment (fluticasone propionate responders (FPR)) had normalized levels (Figure 6a, FPR). Levels of NTRK1 mRNA highly correlated with $I L 13$ mRNA (Figure $\mathbf{6 b}, r=0.75, P=0.0001$ ) and with eosinophil counts in biopsies of EoE patients (Figure $6 c, r=0.46, P=0.006$ ). Accordingly, NTRK1 protein was significantly increased in biopsies from patients with active EoE compared with unaffected individuals (Figure 6d,e). Notably, neither mRNA nor protein levels of NGF were significantly altered in the biopsies from EoE patients; as a positive control, CCL26 mRNA and protein were highly increased (Figure 6f,g). Immunohistochemistry of control and EoE biopsies substantiated our findings by showing positive NTRK1 staining in the cytoplasm and membrane of epithelial cells in the basal layer in active EoE samples but not in control unaffected samples (Figure 6h). Notably, NGF showed diffuse staining throughout the esophageal epithelium independent of the disease activity. Therefore, whereas the ligand NGF is constitutively expressed, NTRK1 is induced by IL-13 and during allergic inflammation. Collectively, these data establish a mechanism wherein the receptor, but not the ligand, is the limiting checkpoint in the pathway. Furthermore, we tested the expression level of EGR1, the main transcriptional target of NGF/NTRK1 signaling ${ }^{15}$ and found that both the mRNA and protein levels of EGR1 were significantly increased in the biopsies from patients with active EoE compared with control samples (Figure 6i-k).

\section{Characterization of TE-7 cells stably expressing NTRK1}

In order to further investigate the functional link between IL-13 and NGF signaling, we generated two pools of TE-7 cells stably expressing NTRK1 that allowed us to simultaneously induce IL-13 and NGF/NTRK1 responses. The majority of the cells expressed NTRK1 in the cytoplasm and membrane at comparable levels between cell lines (Figure 7a,b, NTRK1 panel), efficiently responded to NGF by phosphorylating NTRK1, and transduced intracellular signaling leading to ERK1/2 phosphorylation (Figure 7b, pNTRK1 and pERK 1/2 panels). Subsequently, rapid and transient induction of EGR1 was observed, indicating that NTRK1 efficiently elicited a transcriptional response (Figure 7c). Notably, while IL-13 efficiently induced CCL26 expression in both control and NTRK1-expressing cells, it failed to induce EGR1 and EGR3 transcription in control clones. As expected, NGF stimulation had no effect on control TE-7 cells, suggesting that NTRK1 is necessary for the activation of EGR genes. Indeed, a decrease in the level of expression of NTRK1 in stable pools and prevention of NTRK1 induction by IL-13 in control pools significantly diminished EGR1 and EGR3 activation by NGF (Figure 7d,e).

We further hypothesized that inhibition of NTRK1 kinase activity will prevent induction of EGR genes following NGF stimulation. To test this hypothesis, we utilized two tyrosine kinase inhibitors, lestaurtinib (CEP-701) and crizotinib, that were previously reported as efficient blockers of NTRK1 autophosphorylation and downstream signaling. ${ }^{40,41}$ As expected, both drugs dramatically reduced the NGF-mediated increase in EGR1 and EGR3 mRNA (Figure 7f). These data suggest that functional NTRK1 is required for efficient EGRs stimulation in epithelial cells.

\section{NTRK1 and IL-13 synergize in propagating allergic responses}

As a readout of functional interaction between NGF/NTRK1 and IL-13, we measured transcription of several known IL-13 target genes, including CCL26, the molecular driver of eosinophilic infiltration in EoE. We stimulated TE-7 cells with IL-13 and NGF either alone or in combination for a period of $6 \mathrm{~h}$ and quantified mRNA levels of the genes. Exposure to NGF alone had no effect on the level of CCL26, SERPINB4, KITLG, or ID3 mRNA, whereas induction with IL-13 caused increased expression of these genes. In NTRK1-expressing cells, the transcription of CCL26, SERPINB4, and KITLG but not ID3 was synergistically increased by NGF and IL-13 (Figure 8a-d). In agreement with these data, secretion of CCL26 from NTRK1-expressing cells after co-stimulation with IL-13 and NGF was significantly higher compared with either signal alone (Figure 8e). Collectively, these findings demonstrated the potential of synergistic interaction between NGF/NTRK1 and IL-13 in propagating allergic inflammation. 

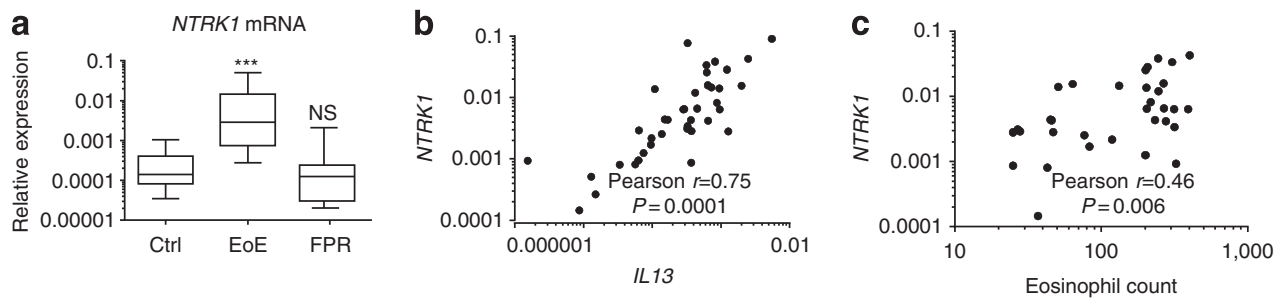
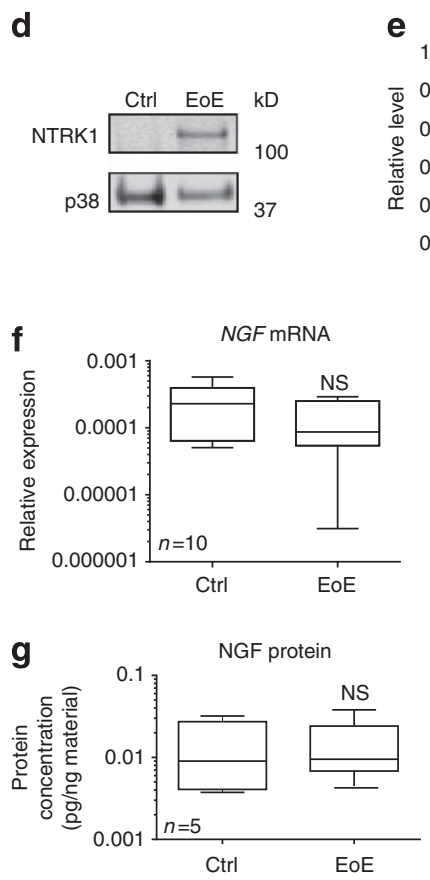

h
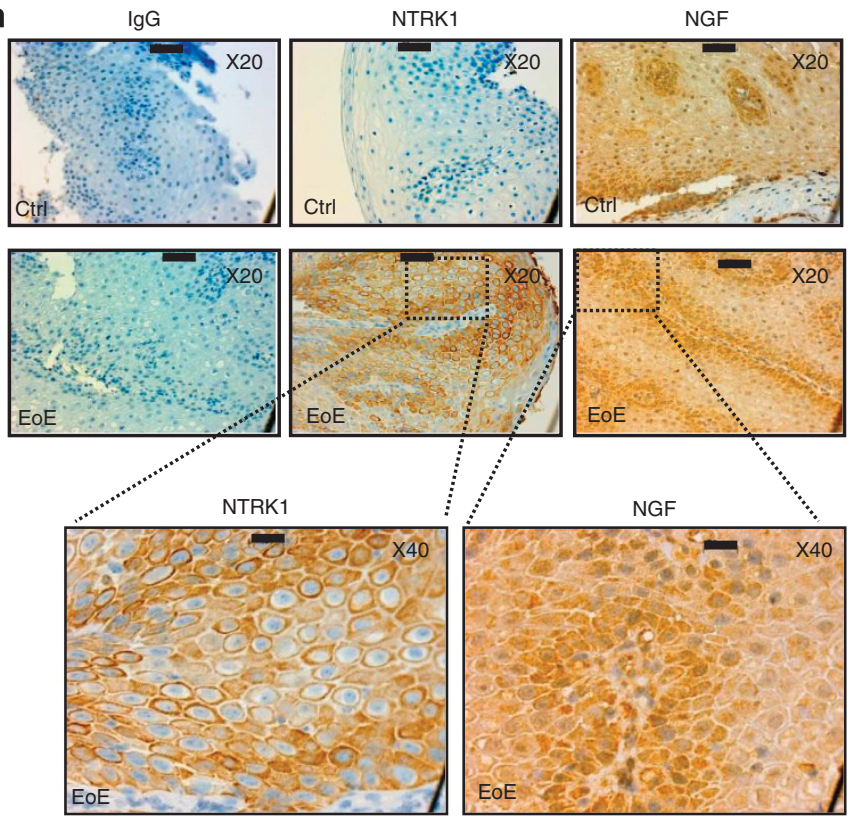
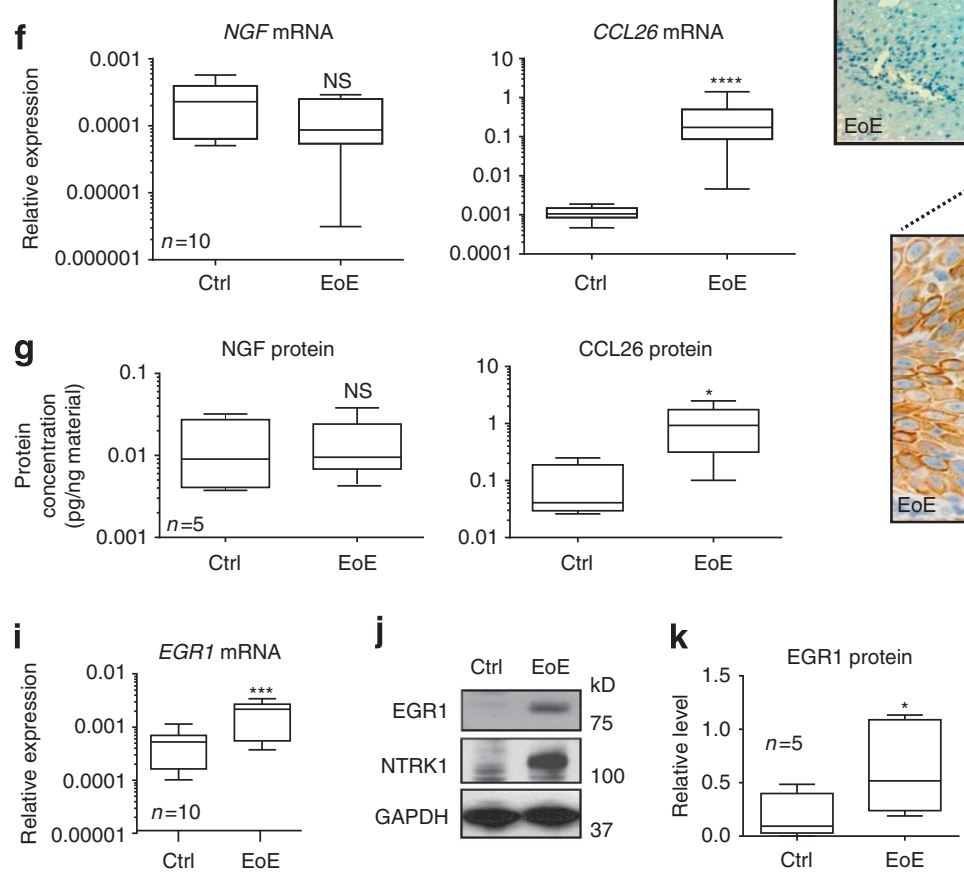

Figure 6 Neurotrophic tyrosine kinase receptor, type 1 (NTRK1), nerve growth factor (NGF), and early growth response protein 1 (EGR1) expression in human allergic inflammation. (a) Real-time PCR (RT-PCR) analysis of NTRK1 in biopsies was performed in unaffected control patients (Ctrl, $n=13$ ), patients with active eosinophilic esophagitis (EoE) $(n=12)$, and EoE patients who responded to swallowed steroid treatment (fluticasone propionate responders (FPR), $n=13$ ); ${ }^{* *} P<0.001$; NS, not significant. (b, c) Graphs show Pearson correlation of NTRK1 expression with IL13 levels and peak eosinophil counts in EoE biopsies. (d) A representative western blotting shows the expression of NTRK1 in a control and EoE biopsy; p38 serves as a loading control. (e) Quantification of NTRK1 in control and active EoE biopsies is shown $(n=5$ each); $P<0.05$. Expression level was normalized to the level of glyceraldehyde 3-phosphate dehydrogenase (GAPDH). (f) RT-PCR analysis of NGF and chemokine (C-C motif) ligand 26 (CCL26) was performed in control and EoE biopsies $\left(n=10\right.$ each); ${ }^{* * *} P<0.0001$; NS, not significant. (g) Amount of NGF and CCL26 protein was measured by enzyme-linked immunosorbent assay in control and active EoE biopsies ( $n=5$ each); ${ }^{*} P<0.05$; NS, not significant. (h) Immunohistochemistry for NTRK1 and NGF in control (Ctrl, no eosinophils present in biopsy) and representative active EoE biopsy (EoE, 199 eosinophils per high-power field is shown. The bar is $20 \mu \mathrm{m}$ for $\times 20$ images (top six panels) and $50 \mu \mathrm{m}$ for $\times 40$ images (bottom two panels). A total of 9 control and 10 active EoE biopsies were stained. (i) EGR1 mRNA was measured by RT-PCR in esophageal biopsies of unaffected control (Ctrl) patients and patients with active EoE ( $n=10$ each). ${ }^{\star \star \star} P<0.001$. (j) A representative western blotting of EGR1 and NTRK1 in control and active EoE biopsies. GAPDH serves as a loading control.

(k) Relative level of EGR1 expression in biopsies is shown ( $n=5$ each); $P<0.05$. Expression level was normalized to the level of $p 38$ protein. For a, $\mathbf{f}$, and $\mathbf{i}$, mean values of gene expression were normalized to the level of $G A P D H$. For $\mathbf{a}, \mathbf{e}, \mathbf{f}, \mathbf{g}$, $\mathbf{i}$, and $\mathbf{k}$, data are presented as box and whiskers plot. IgG, immunoglobulin G.

\section{EGR1 is required for efficient induction of IL-13 target genes}

EGR1 has been previously implicated in IL-13-mediated allergic inflammation and tissue remodeling in mice. ${ }^{16}$ Although we could not detect increased EGR1 expression after IL-13 stimulation (Figure 7c), synergistic induction of several IL-13 target genes by IL-13 and NGF prompted us to test whether EGR1 is required for efficient IL-13 response in esophageal epithelial cells. As TE-7 cells express detectable levels of EGR1 at baseline (Figure 5e), we utilized a small interfering RNA 


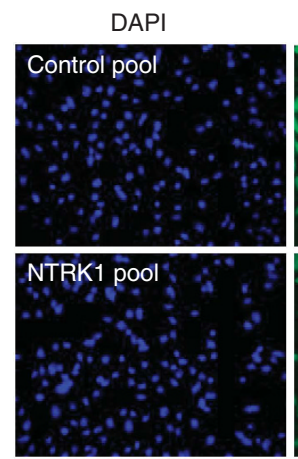

ERK $1 / 2$

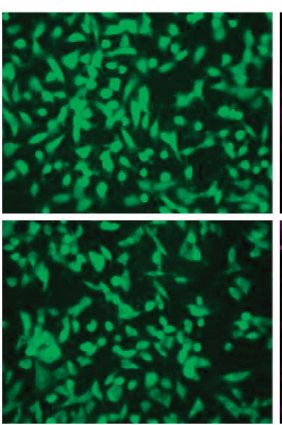

NTRK1

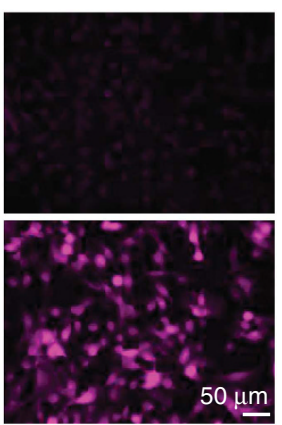

b

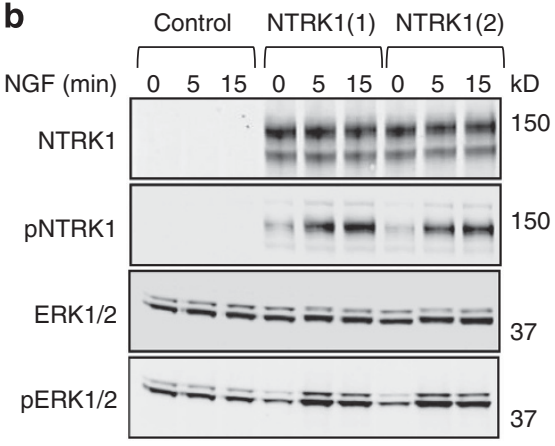

C
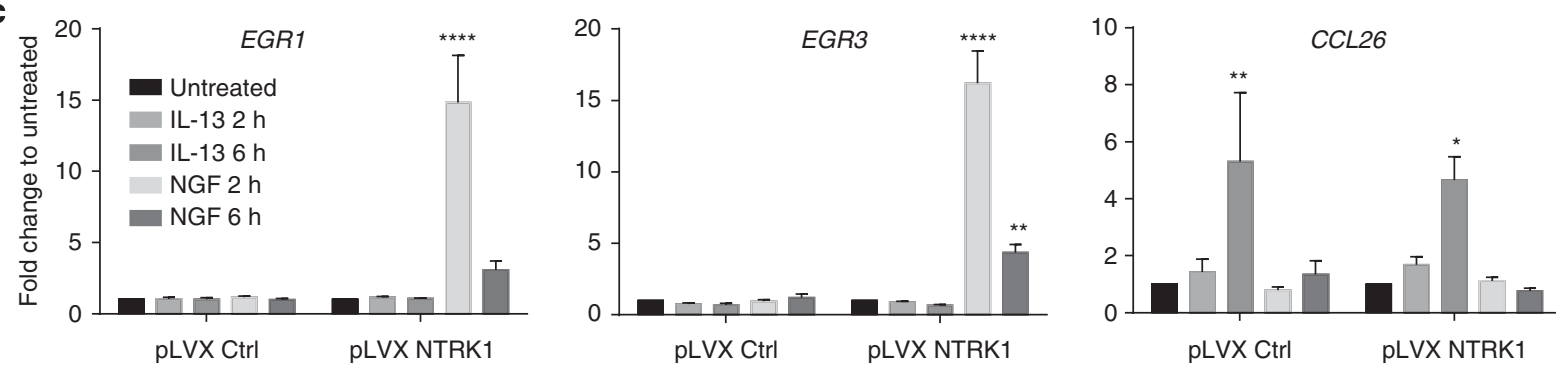

d

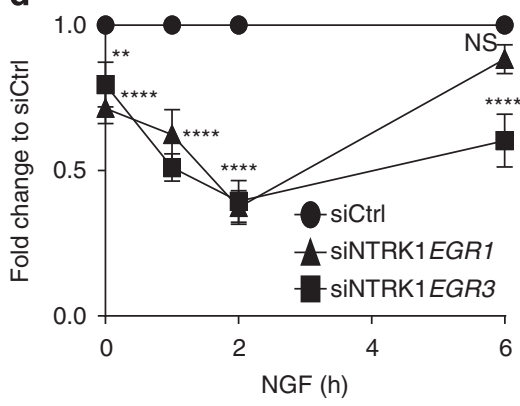

e

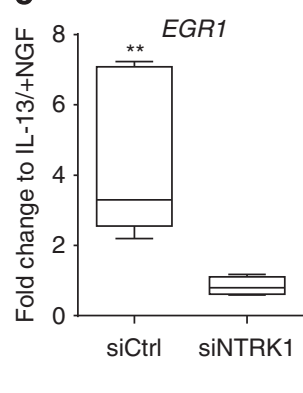

pLVX NTRK1
pLVX Ctrl

\section{$f$}

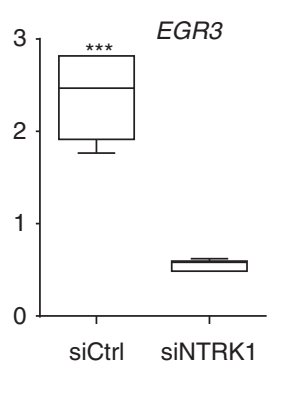

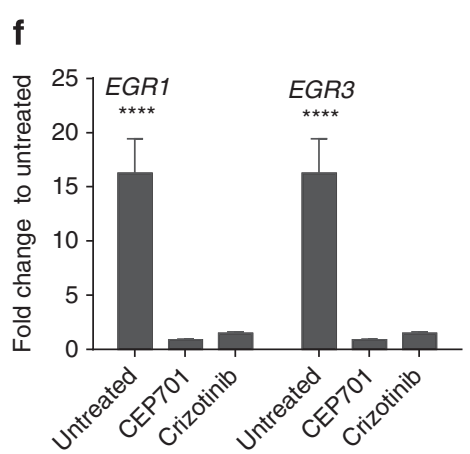

Figure 7 Characterization of TE-7 pools stably overexpressing neurotrophic tyrosine kinase receptor, type 1 (NTRK1). (a) A representative immunofluorescent image of TE-7 cells infected with either empty virus (control pool) or virus expressing NTRK1 (NTRK1 pool) is shown. (b) Western blotting analysis of two independent pools of TE-7 cells stably expressing NTRK1 (NTRK1 (1) and NTRK1 (2)). Cells were treated with nerve growth factor (NGF; $100 \mathrm{ng} \mathrm{ml}^{-1}$ ) for the indicated times. pNTRK1 and pERK1/2 indicate phosphorylated proteins. (c) Effect of NGF and interleukin (IL)-13 stimulation on early growth response protein 1 (EGR1), EGR3, and chemokine (C-C motif) ligand 26 (CCL26) expression was assessed by real-time PCR (RT-PCR) in control (pLVX Ctrl) and NTRK1 stably expressing cells (pLVX NTRK1). (d) Effect of NTRK1 downregulation in TE-7 cells stably expressing NTRK1 on EGR1 and EGR3 induction by NGF was assessed by RT-PCR. Cells were treated with small interfering RNA (siRNA) for $36 \mathrm{~h}$ followed by stimulated with NGF at $10 \mathrm{ng} \mathrm{ml}^{-1}$ for the indicated periods of time. Expression level of EGRs relative to siCtrl-treated cells is shown. Data for three independent experiments are presented; error bars represent s.e. measurements. (e) Effect of decreased expression of NTRK1 in IL-13-pretreated TE-7 control pool on EGR1 and EGR3 induction by NGF was assessed by RT-PCR. Cells were treated with siRNA for $18 \mathrm{~h}$ and induced with IL-13 at $10 \mathrm{ng} \mathrm{ml} \mathrm{ml}^{-1}$ for $24 \mathrm{~h}$ followed by stimulation with NGF at $10 \mathrm{ng} \mathrm{ml}^{-1}$ for $1 \mathrm{~h}$. siCtrl, control siRNA, siNTRK1, siRNA against NTRK1. Data for three independent experiments are presented as box and whiskers plot. (f) Effect of pretreatment of TE-7 cells stably expressing NTRK1 with tyrosine kinase inhibitors on EGR1 and EGR3 induction by NGF was assessed by RT-PCR. Cells were pretreated with the inhibitors at $0.1 \mu \mathrm{m}$ for 15 min following stimulation with NGF at $10 \mathrm{ng} \mathrm{ml}^{-1}$ for $2 \mathrm{~h}$. Data for two independent experiments are shown. ${ }^{\star \star \star \star} P<0.0001,{ }^{\star \star \star} P<0.001,{ }^{\star \star} P<0.01,{ }^{\star} P<0.05$. NS, not significant.

(siRNA) approach to decrease EGR1 expression in TE-7 cells prior to IL-13 stimulation. We efficiently downregulated EGR1, but not EGR3 expression, in cells by about 3-5-fold (Figure 8f, EGR1, EGR3). In TE-7 cells with decreased EGR1 level, the ability of IL-13 to induce the expression of some target genes (CCL26, CDH26, and SERPINB4), but not others (KITLG, NTRK1), was significantly diminished (Figure 8f). These findings implicate EGR1 in the propagation of IL-13-mediated allergic inflammation in human epithelial cells and provide mechanistic insight into synergistic interaction between NGF and IL-13 pathways.

\section{DISCUSSION}

By analyzing the kinetics of gene expression in IL-13stimulated human epithelial cells, we have identified NTRK1, a high-affinity receptor for NGF, as a direct transcriptional and epigenetic target of IL-13. In support of this finding, we showed that NTRK1 was highly induced by IL-13 in epithelial cells, including those derived from several sources (e.g., bronchi and esophagus). This induction was STAT6 dependent and readily detectable as early as $2 \mathrm{~h}$ after IL-13 addition, and the level of NTRK1 decreased as IL-13 was withdrawn (data not shown). In addition, in response to IL-13, the NTRK1 promoter 

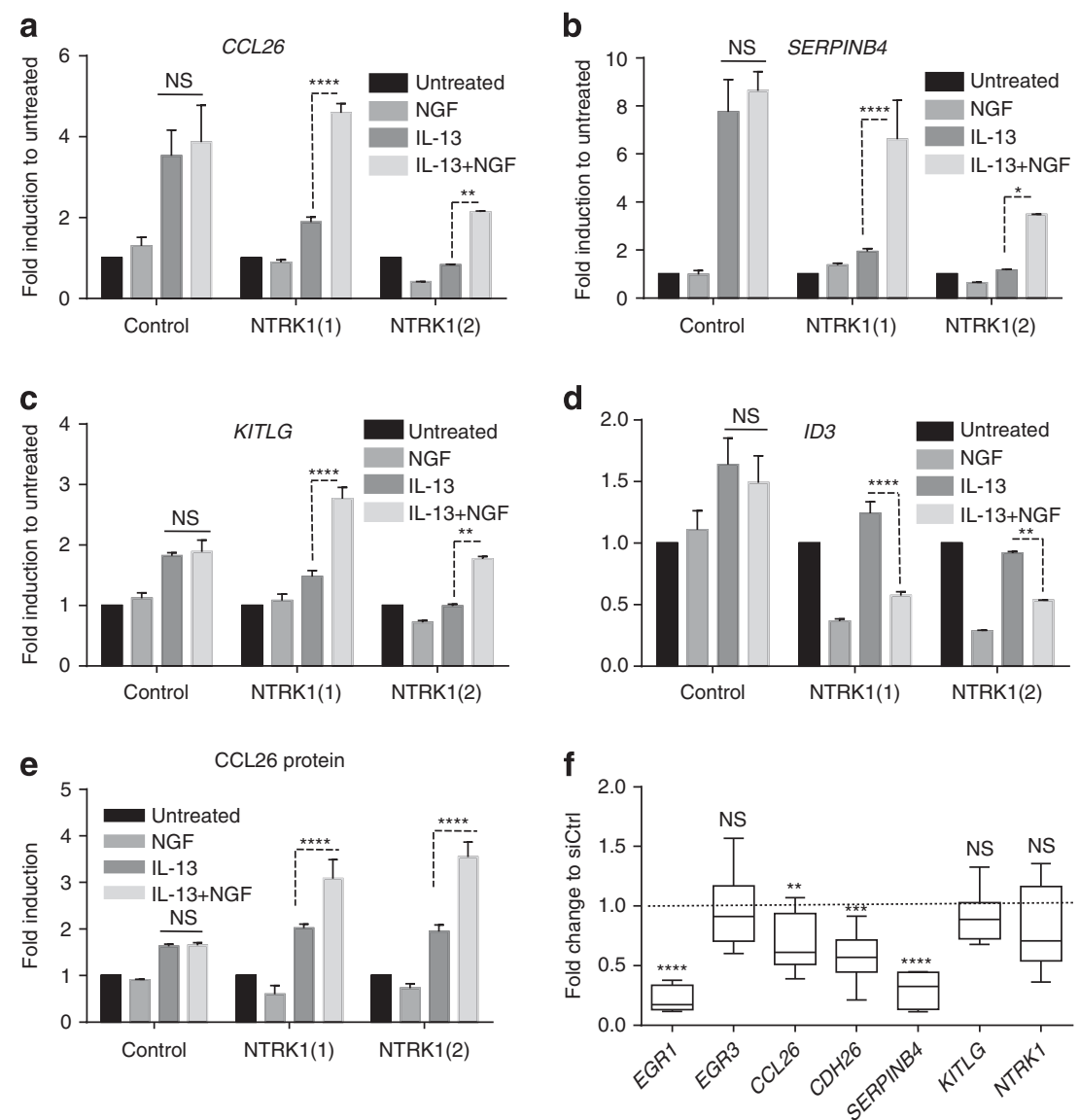

Figure 8 Interleukin (IL)-13 and nerve growth factor/neurotrophic tyrosine kinase receptor, type 1 (NGF/NTRK1) synergistically stimulate chemokine (C-C motif) ligand 26 (CCL26) production. (a-d) Transcriptional levels of CCL26, serpin peptidase inhibitor, clade B, member 4 (SERPINB4), KIT ligand ( $K I T L G)$, and inhibitor of DNA binding 3 (ID3) after IL-13 and/or NGF stimulation of TE-7 cells stably expressing NTRK1 were measured by real-time PCR. Induction was performed for $6 \mathrm{~h}, \mathrm{IL}-13$ concentration was $1 \mathrm{ng} \mathrm{ml}^{-1}$, and NGF was used at $100 \mathrm{ng} \mathrm{ml}^{-1}$. Expression was normalized to the level of GAPDH. (e) CCL26 protein level in cell supernatant was measured by enzyme-linked immunosorbent assay. Cells were stimulated with IL-13 and NGF $\left(100 \mathrm{ng} \mathrm{ml}^{-1}\right)$ for $24 \mathrm{~h}$; mean fold induction relative to untreated cells is shown. Error bars represent s.e.m. for four independent experiments. (f) Relative level of expression of the indicated genes following downregulation of early growth response protein 1 (EGR1) by small interfering RNA (siRNA) in TE-7 cells. Expression of genes in cells transfected with control siRNA (siCtrl) was set as 1 (dotted line). Data for $4-5$ independent experiments are presented as box and whiskers plot; ${ }^{\star \star \star \star} P<0.0001,{ }^{\star \star \star} P<0.001,{ }^{\star \star} P<0.01,{ }^{\star} P<0.05$. NS, not significant.

acquired a set of activating epigenetic marks, including histone $\mathrm{H} 3$ acetylation and trimethylation, as early as $2 \mathrm{~h}$ after IL-13 addition and continued accumulating these marks throughout the stimulation in a STAT6-dependent manner, mirroring the expression pattern of the gene.

Translational studies showed elevated NTRK1 expression in the biopsies of patients with active EoE and that this increased NTRK1 expression normalized after steroid treatment. Moreover, NTRK1 mRNA levels strongly correlated with the markers of disease activity, such as $I L-13$ mRNA level and eosinophil counts. This pattern of expression resembles that of CCL26, a critical eosinophil chemoattractant in the pathogenesis of EoE. ${ }^{19}$ Our in vitro finding that NGF and IL-13 synergistically induce a number of hallmark genes, including CCL26, in conjunction with in vivo data showing dynamic expression of NTRK1 as a function of disease severity collectively suggest that the NGF/NTRK1 and IL-13/STAT6 pathways functionally cooperate in propagating allergic inflammation. We indeed observed increased levels of EGR1, a central transcriptional target of NGF, ${ }^{15}$ in biopsies of patients with active EoE. EGR1 has been previously shown to be induced in lung epithelial cells exposed to dust mite allergen ${ }^{42}$ and to have an important role in the induction of T helper type 2-related chemokines. ${ }^{16} E G R 1$ is rapidly and transiently induced in response to various stimuli, including growth factors or cytokines, ${ }^{43,44}$ mechanical stress, ${ }^{45}$ and hypoxia. ${ }^{46}$ Therefore, we cannot definitively state that EGR1 elevation is solely due to NGF/NTRK1 signaling. Regardless, these findings suggest potential functional interactions of NGF/NTRK1 and IL-13 pathways in the pathogenesis of allergic inflammation.

Unlike mouse models, ${ }^{16,47}$ we could not detect increased level of EGR1 in response to IL-13 stimulation. Yet our findings that decreased EGR1 expression correlated with diminished IL-13 response suggest that EGR1 synergizes with IL-13 in induction of early target genes presumably by interacting with STAT6. Cooperative interaction of EGR1 with other 
transcription factors, such as NF of activated T cells and NF- $\kappa \mathrm{B}$ has been previously reported in T cells. ${ }^{48}$ Similarly, synergistic interaction between STAT6 and NF- $\kappa \mathrm{B}$ in IL-4-induced transcription has been shown. ${ }^{49}$ Moreover, EGR1 has been implicated in the formation of chromatin loops required for its synergistic interaction with other transcriptional factors, ${ }^{50}$ substantiating the potential role of epigenetics in allergic transcriptional response. Notably, EGR1 was required for efficient IL-13 induction of some but not all genes, suggesting that other factors are required for synergistic activation of target genes by IL-13 and NGF during the propagation of allergic inflammation.

Epigenetics is considered as a possible mechanism involved in the development of many disorders, including allergic diseases. ${ }^{51,52}$ The most common epigenetic mechanisms include DNA methylation, histone modifications, and noncoding RNAs, all of which can affect gene transcription through effects on DNA structure and inducing gene silencing. We have previously demonstrated that these mechanisms are involved in IL-13-mediated allergic inflammation. ${ }^{53-55}$ Accordingly, IL-13-mediated NTRK1 induction was accompanied by increased levels of activating epigenetic modifications. Notably, we have found high levels of activating marks in the promoter of NTRK1 prior to IL-13 stimulation, suggesting that NTRK1 is epigenetically poised for induction. ${ }^{56-58}$ Similarly, elevated levels of histone acetylation were observed in the CCL26 promoter, collectively suggesting that epigenetic poising may be a common mechanism of induction for early IL-13 targets. Interestingly, the level of the H3K4me3 mark, which is commonly present at the promoters of transcribed genes, ${ }^{59}$ was relatively low on the CCL26 promoter compared with NTRK1. A low level of H3K4me3 has been observed previously in $\sim 20 \%$ of the expressed genes, and the percentage of genes with the H3K4me3 mark was not increased following cell activation. ${ }^{56}$ The low level of $\mathrm{H} 3 \mathrm{~K} 4 \mathrm{me} 3$ in the promoter of the CCL26 gene may reflect a more "enhancer-like" signature of this region, which is consistent with the known signature of active enhancers (low H3K4me3, high H3K27Ac) ${ }^{60}$ Alternatively, it is possible that the peak of $\mathrm{H} 3 \mathrm{~K} 4 \mathrm{me} 3$ in the promoter of CCL26 is shifted relatively to other histone marks and therefore is not detected by RT-PCR. Performing chromatin immuneprecipitation (ChIP)-sequencing experiments should differentiate between these possibilities. The ultimate goal of future studies will be the comprehensive characterization of the IL-13-mediated epigenome by ChIPsequencing and utilizing this knowledge in developing new diagnostic and therapeutic strategies in allergy.

Among the 90 RTKs identified in the human genome, ${ }^{36}$ NTRK1 was the only one induced both transcriptionally and epigenetically by IL-13 in esophageal epithelial cells. Remarkably, we did not observe significant changes in the NGF level in esophageal biopsies, where it was detected independent of the disease status. Transcriptional regulation of NTRKs has been widely investigated; ${ }^{61}$ however, to the best of our knowledge, these data are the first example of regulating NTRK1 signaling by modulating the level of the receptor rather than the ligand in

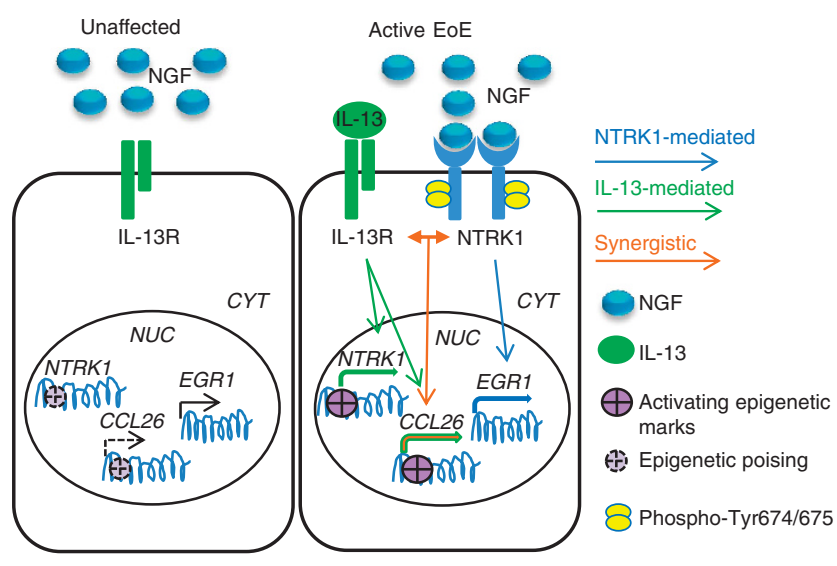

Figure 9 Model for propagation of interleukin (IL)-13-mediated allergic inflammation in epithelial cells. Prior to IL-13 stimulation (represented by unaffected cell), promoters of chemokine (C-C motif) ligand 26 (CCL26) and neurotrophic tyrosine kinase receptor, type 1 (NTRK1) are epigenetically poised for activation (small light purple circles with broken lines), but genes are either not transcribed (NTRK1) or transcribed at a low level (CCL26, broken arrow). Early response gene protein 1 (EGR1) is transcribed at a detectable level (represented by solid arrow). Nerve growth factor (NGF) is present in the environment but does not elicit a transcriptional response. During allergic inflammation (exemplified by active eosinophilic esophagitis (EoE)), IL-13 initially induces epigenetic and transcriptional responses of its targets, as shown for CCL26 and NTRK1 (large purple circles with solid lines and green arrows), and increased expression of NTRK1. Subsequently, NGF binds to NTRK1 and elicits signal transduction (illustrated by phosphorylation of NTRK1 on kinase domain (yellow ovals)), which leads to an NGF/NTRK1-mediated transcriptional response (e.g., induction of EGR1, blue arrow), as well as a synergistic effects on transcription of key inflammatory genes (orange arrows, exemplified by CCL26). IL-13R, IL-13 receptor; NUC, nucleus; CYT, cytoplasm.

human epithelial cells. One previous study described downregulation of NTRK1, but not NGF, in the cerebellum of rat pups exposed to ethanol. ${ }^{62}$ On the basis of our findings, we propose that an NTRK1/IL-13 axis is involved in the propagation of allergic inflammation (Figure 9). In this model, early transcriptional targets of IL-13 in epithelial cells are epigenetically poised for expression, as evident from the presence of activating epigenetic marks on the promoters of genes prior to IL-13 stimulation. IL-13 causes increased expression of NTRK1 together with other early transcriptional targets (CCL26) accompanied by augmenting epigenetic changes in the promoters of these genes. Subsequently, NTRK1 becomes activated by the extracellular pool of NGF molecules, which in turn leads to NTRK1-mediated transcriptional responses (e.g., increased EGR1), as well as synergistic interaction with IL-13 in eliciting transcription and secretion of CCL26 and other mediators of the allergic response.

In summary, we identify NTRK1 as an early epigenetic and transcriptional target of IL-13 in human epithelial cells. We provide evidence that NTRK1, but not its ligand NGF, is dramatically upregulated in the biopsies of patients with active EoE, establishing a mechanism wherein the receptor, but not the ligand, is the limiting checkpoint in the pathway. We demonstrate synergistic induction of the critical allergic 
mediator CCL2 6 by IL-13 and NGF/NTRK1 signaling, supporting the role of NTRK1 in the pathogenesis of mucosal allergic inflammation.

Our finding that NTRK1 is highly increased in human esophageal biopsies from EoE patients and synergizes with IL-13 in propagating allergic inflammation provides the rationale for pharmacological targeting of NTRK1 signaling. The increasingly recognized link between NTRK1 signaling and cancer progression, ${ }^{40,63-65}$ as well as the critical role of NTRK1 in pain sensitivity, ${ }^{32,66,67}$ has stimulated the development of highly specific NTRK1 inhibitors, such as AR-786, AR-256, and AR-618. ${ }^{68}$ Additionally, the wide range of existing tyrosine kinase inhibitors specific to a number of protein kinases, including NTRK1, such as lestaurtinib (CEP-701) and crizotinib used in our study, can be utilized to block NTRK1 activity. Some of these inhibitors have been approved for use in human subjects primarily for cancer treatment. ${ }^{41,69}$ Importantly, because NTRK1 is the only protein tyrosine kinase dramatically induced by IL-13 in epithelial cells, even the use of tyrosine kinase inhibitors not specifically developed for NTRK1 may be beneficial for decreasing allergic inflammation. Our experiments provide initial support for the feasibility of this approach.

\section{METHODS}

Cell culture and treatment. Primary esophageal epithelial cells were cultured as previously described. ${ }^{10}$ The squamous esophageal epithelial cell line TE-7 (a kind gift of Dr Hainault, France), which was originally selected from human esophageal tumors, ${ }^{17}$ was maintained in RPMI-1640 medium (Invitrogen, Carlsbad, CA) supplemented with $5 \%$ fetal calf serum (FCS). The esophageal epithelial cell line (hTERT-immortalized EPC2 line) was a kind gift from Dr Anil Rustgi (University of Pennsylvania). EPC2 cell monolayers were grown on $0.4-\mu \mathrm{m}$ pore-size polyester permeable supports (Corning Incorporated, Corning, NY) in keratinocyte serum-free media (Life Technologies, Grand Island, NY). Once confluent, medium was switched to high-calcium $\left(\left[\mathrm{Ca}^{2+}\right]=1.8 \mathrm{~mm}\right)$ keratinocyte serum-free media for an additional 3-5 days. Epithelial differentiation was then induced by removing culture media from the inner chamber of the permeable support and maintaining the esophageal epithelial cells for 5-7 days at the air-liquid interface. Normal human primary bronchial epithelial cells were purchased from Lonza (Walkersville, MD, CC-2540) and cultured as previously described. ${ }^{70}$ IL-13 (Peprotech, Rocky Hill, NJ) and NGF (human $\beta$-NGF; Cell Signaling Technology, Danvers, MA) were added to the culture media at the indicated concentrations of 1 , 10 , or $100 \mathrm{ng} \mathrm{ml}^{-1}$ for the indicated periods of time. Tyrosine kinase inhibitors crizotinib (PZ0191) and lestaurtinib (CEP-701 hydrate, C7869) were purchased from Sigma-Aldrich (Sigma-Aldrich, St Louis, $\mathrm{MO}$ ) and were dissolved in dimethyl sulfoxide to stock concentration of $10 \mathrm{~mm}$. Cells were pretreated with the inhibitors at $0.1 \mu \mathrm{M}$ for $15 \mathrm{~min}$ prior to stimulation with NGF at $10 \mathrm{ng} \mathrm{ml}^{-1}$ for $2 \mathrm{~h}$.

Antibodies. Rabbit antibodies against TrkA (14G6) (no. 2508), phospho-TrkA (Tyr674/675)/TrkB (Tyr706/707) (no. C50F3); phospho-p44/42 MAPK (ERK1/2) (Thr202/Tyr204) (no. 9101), p44/ 42 MAPK (ERK1/2) (137F5) (no. 4695); phospho-STAT6 (Tyr641) (C11A12); EGR1 (44D5) (no. 4154); EGR3 (no. 2559), p38 MAPK (D13E1) XP (no. 8690); mouse monoclonal antibody against p44/42 MAPK (Erk1/2) (3A7, no. 9107), and anti-rabbit immunoglobulin G, HRP-linked (no. 7074) were purchased from Cell Signaling Technology. STAT6 (S-20, sc-621) was from Santa Cruz Biotechnology (Dallas, TX). Monoclonal anti-GAPDH (glyceraldehyde 3-phosphate dehydrogenase)-peroxidase clone GAPDH-71.1 (no. G9295) was from
Sigma-Aldrich. For ChIP, H3K4me3 (ab8580), H3K9Ac (ab10812), and H3K27Ac (ab4279) were from Abcam (Cambridge, MA). Some ChIP experiments were performed with H3K27Ac antibody from Diagenode (pAb-196-050, Denville, NJ) and H3K4me3 antibody from Millipore (17-614, Billerica, MA). For immunofluorescence, goat antirabbit Alexa Fluor 647 (A-21244) and goat anti-mouse Alexa Fluor 488 (A11029) were from Invitrogen.

STAT6 gene silencing in TE-7 cells. Lentiviral shRNA vector against STAT6 (MISSION shRNA, Sigma-Aldrich, clone ID NM_003153.33231s1c1, TRCN0000019409) was used to produce lentiviral particles by the Cincinnati Children's Hospital Medical Center (CCHMC) Lenti-shRNA Library Core. A vector that targets no known mammalian genes was used as a control (SHC002 SIGMA MISSION pLKO.1-puro Non-Mammalian shRNA Control). TE-7 cells were grown in RPMI medium with 5\% FCS and infected on six-well plates in the presence of polybrene at $5 \mu \mathrm{g} \mathrm{ml}^{-1}$ by centrifuging the plate at $2,000 \mathrm{~g}$ for $1 \mathrm{~h}$ at room temperature (RT). The following day, puromycin selection was applied at $2 \mu \mathrm{g} \mathrm{ml}^{-1}$ for at least 7 days. shSTAT6 stably infected cells were maintained in puromycin at $1 \mu \mathrm{g} \mathrm{ml}^{-1}$. Puromycin was removed $24 \mathrm{~h}$ prior to the start of experiments.

EGR1 and NTRK1 gene silencing in TE-7 cells. For EGR1 silencing, TE-7 cells were grown on 12-well plates in RPMI with 1\% FCS medium and transfected with SMART pool ON-TARGET plus EGR1 siRNA (L-006526-00-0005; GE Dharmacon, Lafayette, CO) using Dharmafect 1 transfection reagent (GE Dharmacon). Final siRNA concentration was $30 \mathrm{~nm}$. Following $36 \mathrm{~h}$ of incubation, cells were stimulated with IL-13 at $1 \mathrm{ng} \mathrm{ml}^{-1}$ for the indicated periods of time without medium change. For NTRK1 silencing, pLVX control and NTRK1 stable pools were grown in RPMI medium with 5\% FCS. SMART pool ON-TARGET plus NTRK1 siRNA (L-003159-00-0005, GE Dharmacon) was used at final concentration of $30 \mathrm{~nm}$. For the control pool, silencing was performed for $18 \mathrm{~h}$ prior to induction with IL-13 at $100 \mathrm{ng} \mathrm{ml}^{-1}$ for $24 \mathrm{~h}$ followed by NGF stimulation. For the NTRK1 pools, silencing was performed for $36 \mathrm{~h}$ prior to NGF stimulation.

Preparation of stable pools of TE-7 cells expressing NTRK1. Homo sapiens neurotrophic tyrosine kinase, receptor, type 1 (NTRK1), transcript variant 1, mRNA (NM_001012331.1) was amplified from the pCMV-Sport6 vector (MHS1010-202736585, clone ID 5200930; Fisher Scientific, Pittsburgh, PA) and cloned between EcoRI and XbaI sites in pLVX-IRES-Puro vector using the In-fusion method (Clontech, Mountain View, CA). Lentiviral particles were produced by transfecting HEK 293T cells according to the protocol recommended by Clontech. TE-7 cells were grown in RPMI medium with 5\% FCS and infected on six-well plates in the presence of polybrene at $5 \mu \mathrm{g} \mathrm{ml}^{-1}$ by centrifuging the plate at $2,000 \mathrm{~g}$ for $1 \mathrm{~h}$ at RT. The following day, puromycin selection was applied at $1 \mu \mathrm{g} \mathrm{ml}^{-1}$ for at least 7 days. NTRK1 stably infected cells were maintained in puromycin at $1 \mu \mathrm{g} \mathrm{ml}^{-1}$. Puromycin was removed $24 \mathrm{~h}$ prior to the start of experiments. Two independent transductions were performed to generate NTRK1 (1) and NTRK1 (2) pools. Expression levels were validated by western blotting and immunofluorescence analysis.

Immunofluorescence of TE-7 cells. For immunofluorescence, pLVX Control and NTRK1 pools cells were grown on round cover slips placed in the well of the six-well plate. Cells were fixed with cold methanol for $10 \mathrm{~min}$ on ice and washed three times with phosphatebuffered saline (PBS) for 5 min each. Blocking was performed in 10\% goat serum for $30 \mathrm{~min}$ at RT, and cover slips were incubated with primary antibodies against NTRK1 (rabbit) and ERK1/2 (mouse) at 1/ 250 dilution for $2 \mathrm{~h}$ at RT. After three additional washes with PBS, secondary antibodies goat anti-rabbit Alexa Fluor 647 and goat antimouse Alexa Fluor 488 were added for $1 \mathrm{~h}$ at RT at 1/500 dilution. Cells were washed three times with PBS with 4',6-diamidino-2-phenylindole (D3571, Molecular Probes, Life Technologies, Grand Island, NY) 
added to the first wash at $0.5 \mu \mathrm{g} \mathrm{ml}^{-1}$ for $20 \mathrm{~min}$ at RT. Cover slips were mounted on the polysine microscope slides on Prolong Gold mounting medium (P36930, Molecular Probes, Life Technology). Images were acquired using an Apotome upright confocal microscope (Zeiss, Thornwood, NY) under the same settings.

mRNA extraction, quantitative RT-PCR, and RNA-sequencing analysis. Total RNA was isolated from cells with the RNeasy mini kit (Qiagen, Valencia, CA) according to the manufacturer's protocol. For RNA-sequencing experiments, RNA was treated with On-Column DNase Digestion kit (Qiagen) according to the supplied protocol. cDNA was synthesized with the iScript synthesis kit (Bio-Rad, Hercules, CA). RT-PCR was performed using a 7900HT Fast Real-Time PCR system from Applied Biosystems (Life Technologies) with FastStart Universal SYBR Green Master mix (Roche Diagnostics, Indianapolis, IN). Next-Generation RNA-sequencing was performed by the CCHMC Genetic Variation and Gene Discovery Core Facility using the Illumina TruSeq kits and sequenced on the Illumina HiSeq2000. For RNA-sequencing analysis, Fastq files from the Illumina pipeline were aligned by TopHat ${ }^{71}$ with $-\mathrm{T}$ and $-\mathrm{G}$ parameters (http:// tophat.cbcb.umd.edu/manual.html). RefSeq annotation from the University of California Santa Cruz (UCSC) genome browser ${ }^{72}$ for hg19 genome was used. The $-\mathrm{T}$ parameter is used to align reads to the human transcriptome only, and the $-G$ parameter is used to provide transcriptome annotation. To estimate the difference between the treated and untreated experiments, the differential expression analysis for sequence count data (DESeq) package was applied. ${ }^{18}$ For heat map generation, Cluster 3.0 was used for clustering data using Euclidean distance with average linkage (http://bonsai.hgc.jp/ mdehoon/software/cluster/software.htm). For visualization, the Java Treeview program was used (http://jtreeview.sourceforge.net/). All the genes expressed below RPKM 1 in IL-13-treated cells were excluded from the analysis. RNA-sequencing data files were uploaded to the GEO database under the accession number GSE57637.

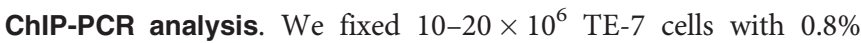
formaldehyde by adding $1 \mathrm{ml}$ of $10 \times$ fixation buffer $(50 \mathrm{~mm}$ Hepes$\mathrm{KOH}, \mathrm{pH} 7.5 ; 100 \mathrm{~mm} \mathrm{NaCl} ; 1 \mathrm{~mm}$ EDTA; $0.5 \mathrm{~mm}$ EGTA; 8\% formaldehyde) to $9 \mathrm{ml}$ of growth medium for $8 \mathrm{~min}$ at RT with shaking. The reaction was stopped by adding glycine to a final concentration of $125 \mathrm{~mm}$ for an additional $5 \mathrm{~min}$. After washing with PBS, pellets were frozen at $-80^{\circ} \mathrm{C}$ for at least overnight. Nuclei were prepared by re-suspending pellets in $1 \mathrm{ml}$ of L1 buffer (50 mm Hepes-KOH, pH 7.5; $140 \mathrm{~mm} \mathrm{NaCl} ; 1$ mм EDTA; $10 \%$ glycerol; 0.5\% NP-40; 0.25\% Triton $\mathrm{X}-100)$ and incubated at $4{ }^{\circ} \mathrm{C}$ for $10 \mathrm{~min}$. Nuclei were pelleted and resuspended in $1 \mathrm{ml}$ of L2 buffer ( $10 \mathrm{~mm}$ Tris- $\mathrm{HCl}, \mathrm{pH} 8.0 ; 200 \mathrm{~mm} \mathrm{NaCl}$; 1 mM EDTA, pH 8.0; 0.5 mm EGTA, pH 8.0) and rotated for 10 min at RT. Nuclei were briefly washed with sonication buffer (Tris-EDTA (TE) buffer $+0.1 \%$ sodium dodecyl sulfate) and re-suspended in $1 \mathrm{ml}$ of sonication buffer. All buffers were supplemented with complete EDTA-free protease inhibitors (Roche Diagnostics). Sonication was performed by a Covaris S220 focused ultrasonicator (Covaris, Woburn, MA) at 175 W Peak Incident Power, 10\% output, 200 bursts for $10 \mathrm{~min}$ in $12 \times 12-\mathrm{mm}^{2}$, round-bottom glass tubes. Efficient DNA fragmentation was verified by agarose gel electrophoresis. ChIP was performed by SX-8G IP-Star Automated System (Diagenode) in radioimmunoprecipitation assay buffer (TE $+0.1 \%$ sodium dodecyl sulfate, $1 \%$ Triton X-100, $150 \mathrm{~mm} \mathrm{NaCl}, 0.1 \%$ sodium deoxycholate) following the protocol of the manufacturer with $2-4 \mu \mathrm{g}$ of the indicated antibodies (see "Antibodies" section). The levels of histone modifications on the promoters were assessed by Prime Time Taqman qPCR assay (Integrated DNA Technologies, Coralville, IA) and normalized to the levels of modifications in the input sample.

Western blotting. Proteins from cell cultures were extracted with radio-immunoprecipitation assay buffer $(50 \mathrm{~mm}$ Tris- $\mathrm{HCl}$ $\mathrm{pH} 8 ; 150 \mathrm{~mm} \mathrm{NaCl} ; 1 \%$ Igepal; $0.5 \%$ sodium deoxycholate; $0.1 \%$ sodium dodecyl sulfate, $1 \mathrm{~mm}$ EGTA, protease and phosphatase inhibitors), loaded on a $4-12 \%$ gel (Invitrogen), and subjected to western blotting analysis with the indicated antibodies.

Procuring and processing of esophageal biopsies. This study was performed with the approval of the CCHMC Institutional Review Board. Informed consent was obtained from patients or their legal guardians to donate tissue samples for research and to have their clinical information entered into the Cincinnati Center for Eosinophilic Disorders database. Patients with no history of EoE or other eosinophilic gastrointestinal disorders and with a current biopsy indicating 0 eosinophils/ $\times 400$ high-power field $(\mathrm{HPF})$ in the distal esophagus and taking no form of glucocorticoid treatment at the time of biopsy served as normal controls. Patients with active EoE were defined as those having $\geqslant 15$ eosinophils/HPF at the time of biopsy and not receiving swallowed glucocorticoid or diet treatment at the time of endoscopy. FPR had a history of EoE, but their current distal esophageal biopsy count was 0 or 1 eosinophil/HPF, and they were receiving swallowed FP at the time of endoscopy. Total RNA was isolated from distal esophageal biopsy specimens using the miRNeasy kit (Qiagen) according to the manufacturer's protocol. For RT-PCR analysis, total RNA (500 ng) was used to synthesize cDNA using Superscript II Reverse Transcriptase (Invitrogen) or iScript (Bio-Rad) using the protocols suggested by the manufacturer. For western blotting, esophageal biopsy protein lysates were prepared on ice by sonication in M-PER reagent (Thermo Fisher Scientific, Waltham, MA) supplemented with $1 \times$ protease inhibitor cocktail (Roche Diagnostics) according to the manufacturer's protocol, and soluble proteins were isolated by centrifugation at $10,000 \mathrm{~g}$ for $10 \mathrm{~min}$ at $4{ }^{\circ} \mathrm{C}$. Immunohistochemical staining of biopsies was performed by the Pathology Research Core at CCHMC. Images were acquired using an Apotome upright confocal microscope (Zeiss).

The enzyme-linked immunosorbent assay (ELISA) for CCL26 and NGF in cells and biopsies. For detection of secreted CCL26, cells were seeded on 12-well plates in RPMI medium containing 5\% FCS. After $24 \mathrm{~h}$, the medium was changed, and cells were allowed to grow for an additional $24 \mathrm{~h}$ prior to stimulation with IL-13 and/or NGF at a final concentration of $100 \mathrm{ng} \mathrm{ml}^{-1}$ for $24 \mathrm{~h}$. At the time of the stimulation, cells were $50 \%$ confluent. $\mathrm{NaCl}$ was added to the supernatant of epithelial cells to a final concentration of $100 \mathrm{~mm}$ prior to collection. For detecting CCL26 and NGF in biopsies, esophageal biopsies were washed twice with $0.5 \mathrm{ml}$ of PBS and sonicated for $2 \mathrm{~min}$ in $130 \mu \mathrm{l}$ of SuperB buffer (Covaris) with addition of EDTA and protease and phosphatase inhibitors in a microTUBE in a S220 sonicator. Sonication conditions were used as recommended by the manufacturer (10\% output, 70 W Peak Incident Power, 200 cycles per burst). Samples were spun down at $10,000 \mathrm{~g}$ for $15 \mathrm{~min}$, and the supernatant was collected for analysis. The bicinchoninic acid protein assay was used to determine the protein concentration of each sample (Thermo Fisher Scientific). Samples were diluted 1:4 in PBS prior to ELISA. Some samples were also used for western blotting. Human CCL26/Eotaxin-3 and NGF DuoSet ELISA kits were used according to the manufacturer's recommendations ( $\& \& D$ Systems, Minneapolis, MN).

Statistical analysis. Statistical analysis was performed using the Prism 6.0 software (GraphPad Software Inc., La Jolla, CA). Comparison among groups was done by the Kruskal-Wallis and analysis of variance tests; $t$-test was applied for comparing two groups of data. A statistical probability of $P<0.05$ was considered significant.

SUPPLEMENTARY MATERIAL is linked to the online version of the paper at http://www.nature.com/mi

\section{ACKNOWLEDGMENTS}

This work was funded by the NIAID (R01AI083450 and R37AI045898), the CURED (Campaign Urging Research for Eosinophilic Disease) Foundation, the Food Allergy Research \& Education (FARE), and the Buckeye Foundation. We thank Shawna Hottinger for editorial assistance; the 
Cincinnati Digestive Health Center Integrative Morphology Core for tissue processing, sectioning, histology, and immunohistochemical staining (NIH P30 DK078392); and Betsy DiPasquale for assistance with immunohistochemical stains.

\section{DISCLOSURE}

M.H.C. is a consultant for Meritage Pharma, Novartis, Receptos, Regeneron, and Aptalis. M.E.R. is a consultant for Immune Pharmaceuticals, Celsus Therapeutics, and Receptos and has an equity interest in each of these; M.E.R. has royalties from reslizumab, a drug being developed by Teva Pharmaceuticals. M.E.R. is an inventor of several patents, owned by Cincinnati Children's, and a set of these patents, related to molecular diagnostics, has been licensed to Diagnovus, LLC. The other authors declared no conflict to interest.

c) 2015 Society for Mucosal Immunology

\section{REFERENCES}

1. Brightling, C.E., Saha, S. \& Hollins, F. Interleukin-13: prospects for new treatments. Clin. Exp. Allergy 40, 42-49 (2010).

2. Abonia, J.P. \& Rothenberg, M.E. Eosinophilic esophagitis: rapidly advancing insights. Annu. Rev. Med. 63, 421-434 (2012).

3. Zheng, T., Oh, M.H., Oh, S.Y., Schroeder, J.T., Glick, A.B. \& Zhu, Z. Transgenic expression of interleukin-13 in the skin induces a pruritic dermatitis and skin remodeling. J. Invest. Dermatol. 129, 742-751 (2009).

4. Lee, J.H. et al. Interleukin-13 induces dramatically different transcriptional programs in three human airway cell types. Am. J. Respir. Cell Mol. Biol. 25 , 474-485 (2001).

5. Kuperman, D., Schofield, B., Wills-Karp, M. \& Grusby, M.J. Signal transducer and activator of transcription factor 6 (Stat6)-deficient mice are protected from antigen-induced airway hyperresponsiveness and mucus production. J. Exp. Med. 187, 939-948 (1998).

6. Kuperman, D.A. et al. Direct effects of interleukin-13 on epithelial cells cause airway hyperreactivity and mucus overproduction in asthma. Nat. Med. 8, 885-889 (2002).

7. Mishra, A. \& Rothenberg, M.E. Intratracheal IL-13 induces eosinophilic esophagitis by an IL-5, eotaxin-1, and STAT6-dependent mechanism. Gastroenterology 125, 1419-1427 (2003).

8. Blanchard, C., Durual, S., Estienne, M., Emami, S., Vasseur, S. \& Cuber, J.C. Eotaxin-3/CCL26 gene expression in intestinal epithelial cells is upregulated by interleukin- 4 and interleukin- 13 via the signal transducer and activator of transcription 6. Int. J. Biochem. Cell Biol. 37, 2559-2573 (2005).

9. Matsukura, S. et al. Interleukin-13 upregulates eotaxin expression in airway epithelial cells by a STAT6-dependent mechanism. Am. J. Respir. Cell Mol. Biol. 24, 755-761 (2001).

10. Blanchard, C. et al. IL-13 involvement in eosinophilic esophagitis: transcriptome analysis and reversibility with glucocorticoids. J. Allergy Clin. Immunol. 120, 1292-1300 (2007).

11. Levi-Montalcini, R. The nerve growth factor 35 years later. Science 237 , 1154-1162 (1987).

12. Bonini, S. et al. Circulating nerve growth factor levels are increased in humans with allergic diseases and asthma. Proc. Natl. Acad. Sci. USA 93 10955-10960 (1996).

13. Sin, A.Z., Roche, E.M., Togias, A., Lichtenstein, L.M. \& Schroeder, J.T. Nerve growth factor or IL-3 induces more IL-13 production from basophils of allergic subjects than from basophils of nonallergic subjects. J. Allergy Clin. Immunol. 108, 387-393 (2001).

14. Hahn, C., Islamian, A.P., Renz, H. \& Nockher, W.A. Airway epithelial cells produce neurotrophins and promote the survival of eosinophils during allergic airway inflammation. J. Allergy Clin. Immunol. 117, 787-794 (2006).

15. Milbrandt, J. A nerve growth factor-induced gene encodes a possible transcriptional regulatory factor. Science 238, 797-799 (1987).

16. Cho, S.J. et al. Role of early growth response-1 (Egr-1) in interleukin-13induced inflammation and remodeling. J. Biol. Chem. 281, 8161-8168 (2006).

17. Nishihira, T., Hashimoto, Y., Katayama, M., Mori, S. \& Kuroki, T. Molecular and cellular features of esophageal cancer cells. J. Cancer Res. Clin. Oncol. 119, 441-449 (1993).
18. Anders, S. \& Huber, W. Differential expression analysis for sequence count data. Genome Biol. 11, R106 (2010).

19. Blanchard, C. et al. Eotaxin-3 and a uniquely conserved gene-expression profile in eosinophilic esophagitis. J. Clin. Invest. 116, 536-547 (2006).

20. Trengove, M.C. \& Ward, A.C. SOCS proteins in development and disease. Am. J. Clin. Exp. Immunol. 2, 1-29 (2013).

21. Spicer, A.P., Olson, J.S. \& McDonald, J.A. Molecular cloning and characterization of a cDNA encoding the third putative mammalian hyaluronan synthase. J. Biol. Chem. 272, 8957-8961 (1997).

22. HajMohammadi, S. et al. Normal levels of anticoagulant heparan sulfate are not essential for normal hemostasis. J. Clin. Invest. 111, 989-999 (2003).

23. Stone, E.L., Lee, S.H., Ismail, M.N. \& Fukuda, M. Characterization of mice with targeted deletion of the gene encoding core 2 beta1,6-N-acetylglucosaminyltransferase-2. Methods Enzymol. 479, 155-172 (2010).

24. Hata, K. et al. Arid5b facilitates chondrogenesis by recruiting the histone demethylase Phf2 to Sox9-regulated genes. Nat. Commun. 4, 2850 (2013).

25. Malinin, N.L., Boldin, M.P., Kovalenko, A.V. \& Wallach, D. MAP3K-related kinase involved in NF-kappaB induction by TNF, CD95 and IL-1. Nature 385, 540-544 (1997)

26. Kas, K. et al. ESE-3, a novel member of an epithelium-specific ets transcription factor subfamily, demonstrates different target gene specificity from ESE-1. J. Biol. Chem. 275, 2986-2998 (2000).

27. Albino, D. et al. ESE3/EHF controls epithelial cell differentiation and its loss leads to prostate tumors with mesenchymal and stem-like features. Cancer Res. 72, 2889-2900 (2012).

28. Wang, Z.Q. et al. Inhibition of RUNX2 transcriptional activity blocks the proliferation, migration and invasion of epithelial ovarian carcinoma cells. PLoS One 8, e74384 (2013).

29. Golonzhka, O. et al. Ctip2/Bcl11b controls ameloblast formation during mammalian odontogenesis. Proc. Natl. Acad. Sci. USA 106, 4278-4283 (2009).

30. Dillon, S.R. et al. Interleukin 31, a cytokine produced by activated T cells, induces dermatitis in mice. Nat. Immunol. 5, 752-760 (2004).

31. Ip, W.K., Wong, C.K., Li, M.L., Li, P.W., Cheung, P.F. \& Lam, C.W. Interleukin-31 induces cytokine and chemokine production from human bronchial epithelial cells through activation of mitogen-activated protein kinase signalling pathways: implications for the allergic response. Immunology 122, 532-541 (2007).

32. Indo, Y. Molecular basis of congenital insensitivity to pain with anhidrosis (CIPA): mutations and polymorphisms in TRKA (NTRK1) gene encoding the receptor tyrosine kinase for nerve growth factor. Hum Mutat. 18, 462-471 (2001).

33. Freund-Michel, V. \& Frossard, N. The nerve growth factor and its receptors in airway inflammatory diseases. Pharmacol. Ther. 117, 52-76 (2008).

34. Frossard, N., Freund, V. \& Advenier, C. Nerve growth factor and its receptors in asthma and inflammation. Eur. J. Pharmacol. 500, 453-465 (2004).

35. Raap, U. \& Braunstahl, G.J. The role of neurotrophins in the pathophysiology of allergic rhinitis. Curr. Opin. Allergy Clin. Immunol. 10, 8-13 (2010).

36. Manning, G., Whyte, D.B., Martinez, R., Hunter, T. \& Sudarsanam, S. The protein kinase complement of the human genome. Science 298, 1912-1934 (2002)

37. Ernst, J. \& Kellis, M. Discovery and characterization of chromatin states for systematic annotation of the human genome. Nat. Biotechnol. 28, 817-825 (2010).

38. Segal, R.A. et al. Differential utilization of Trk autophosphorylation sites. J. Biol. Chem. 271, 20175-20181 (1996).

39. Ginty, D.D., Glowacka, D., Bader, D.S., Hidaka, H. \& Wagner, J.A. Induction of immediate early genes by $\mathrm{Ca} 2+$ influx requires CAMPdependent protein kinase in PC12 cells. J. Biol. Chem. 266, 17454-17458 (1991).

40. Vaishnavi, A. et al. Oncogenic and drug-sensitive NTRK1 rearrangements in lung cancer. Nat. Med. 19, 1469-1472 (2013).

41. Festuccia, C. et al. Tyrosine kinase inhibitor CEP-701 blocks the NTRK1/ NGF receptor and limits the invasive capability of prostate cancer cells in vitro. Int. J. Oncol. 30, 193-200 (2007). 
42. Vroling, A.B., Jonker, M.J., Breit, T.M., Fokkens, W.J. \& van Drunen, C.M. Comparison of expression profiles induced by dust mite in airway epithelia reveals a common pathway. Allergy 63, 461-467 (2008).

43. Cao, X.M., Guy, G.R., Sukhatme, V.P. \& Tan, Y.H. Regulation of the Egr-1 gene by tumor necrosis factor and interferons in primary human fibroblasts. J. Biol. Chem. 267, 1345-1349 (1992).

44. Hodge, C., Liao, J., Stofega, M., Guan, K., Carter-Su, C. \& Schwartz, J. Growth hormone stimulates phosphorylation and activation of elk-1 and expression of c-fos, egr-1, and junB through activation of extracellular signal-regulated kinases 1 and 2. J. Biol. Chem. 273, 31327-31336 (1998).

45. Morawietz, H. et al. Rapid induction and translocation of Egr-1 in response to mechanical strain in vascular smooth muscle cells. Circ. Res. 84, 678-687 (1999).

46. Yan, S.F. et al. Egr-1, a master switch coordinating upregulation of divergent gene families underlying ischemic stress. Nat. Med. 6, 13551361 (2000).

47. Ingram, J.L. et al. Opposing actions of Stat 1 and Stat6 on IL-13-induced up-regulation of early growth response- 1 and platelet-derived growth factor ligands in pulmonary fibroblasts. J. Immunol. 177, 4141-4148 (2006).

48. Lohoff, M., Giaisi, M., Kohler, R., Casper, B., Krammer, P.H. \& Li-Weber, M. Early growth response protein-1 (Egr-1) is preferentially expressed in T helper type 2 (Th2) cells and is involved in acute transcription of the Th2 cytokine interleukin-4. J. Biol. Chem. 285, 1643-1652 (2010).

49. Shen, C.H. \& Stavnezer, J. Interaction of stat6 and NF-kappaB: direct association and synergistic activation of interleukin-4-induced transcription. Mol. Cell. Biol. 18, 3395-3404 (1998).

50. Inoue, K. \& Negishi, M. Early growth response 1 loops the CYP2B6 promoter for synergistic activation by the distal and proximal nuclear receptors CAR and HNF4alpha. FEBS Lett. 583, 2126-2130 (2009).

51. Lovinsky-Desir, S. \& Miller, R.L. Epigenetics, asthma, and allergic diseases: a review of the latest advancements. Curr. Allergy Asthma Rep. 12, 211-220 (2012).

52. Tezza, G., Mazzei, F. \& Boner, A. Epigenetics of allergy. Early Hum. Dev. 89 (Suppl 1), S20-S21 (2013).

53. Lim, E.J., Lu, T.X., Blanchard, C. \& Rothenberg, M.E. Epigenetic $r$ egulation of the IL-13-induced human eotaxin-3 gene by CREB-binding protein-mediated histone 3 acetylation. J. Biol. Chem. 286, 13193-13204 (2011).

54. Lu, T.X. et al. MiR-375 is downregulated in epithelial cells after IL-13 stimulation and regulates an $\mathrm{L}-13$-induced epithelial transcriptome. Mucosal Immunol. 5, 388-396 (2012).

55. Lim, E. \& Rothenberg, M.E. Demethylation of the human eotaxin-3 gene promoter leads to the elevated expression of eotaxin-3. J. Immunol. 192, 466-474 (2014).

56. Barski, A. et al. Chromatin poises miRNA- and protein-coding genes for expression. Genome Res. 19, 1742-1751 (2009).
57. Zediak, V.P., Johnnidis, J.B., Wherry, E.J. \& Berger, S.L. Cutting edge: persistently open chromatin at effector gene loci in resting memory CD8 + Tcells independent of transcriptional status. J. Immunol. 186, 2705-2709 (2011).

58. Russ, B.E., Prier, J.E., Rao, S. \& Turner, S.J. T cell immunity as a tool for studying epigenetic regulation of cellular differentiation. Front. Genet. 4 218 (2013).

59. Guenther, M.G., Levine, S.S., Boyer, L.A., Jaenisch, R. \& Young, R.A. A chromatin landmark and transcription initiation at most promoters in human cells. Cell 130, 77-88 (2007).

60. Creyghton, M.P. et al. Histone H3K27ac separates active from poised enhancers and predicts developmental state. Proc. Natl. Acad. Sci. USA 107, 21931-21936 (2010).

61. Lei, L. \& Parada, L.F. Transcriptional regulation of Trk family neurotrophin receptors. Cell. Mol. Life Sci. 64, 522-532 (2007).

62. Dohrman, D.P., West, J.R. \& Pantazis, N.J. Ethanol reduces expression of the nerve growth factor receptor, but not nerve growth factor protein levels in the neonatal rat cerebellum. Alcohol. Clin. Exp. Res. 21, 882-893 (1997).

63. Dalal, R. \& Djakiew, D. Molecular characterization of neurotrophin expression and the corresponding tropomyosin receptor kinases (trks) in epithelial and stromal cells of the human prostate. Mol. Cell. Endocrinol. 134, 15-22 (1997).

64. Descamps, S. et al. Expression of nerve growth factor receptors and their prognostic value in human breast cancer. Cancer Res. 61, 4337-4340 (2001).

65. Brodeur, G.M. et al. Expression of TrkA, TrkB and TrkC in human neuroblastomas. J. Neurooncol. 31, 49-55 (1997).

66. Indo, Y. Nerve growth factor and the physiology of pain: lessons from congenital insensitivity to pain with anhidrosis. Clin. Genet. 82, 341-350 (2012).

67. Indo, Y. et al. Mutations in the TRKA/NGF receptor gene in patients with congenital insensitivity to pain with anhidrosis. Nat. Genet. 13, 485-488 (1996).

68. Allosteric Small Molecule Inhibitors of the NGF/TrkA Pathway A New Approach to Treating Inflammatory Pain. http://www.arraybiopharma. com/files/6313/9810/8021/PubAttachment587.pdf. Accessed Date.

69. Madhusudan, S. \& Ganesan, T.S. Tyrosine kinase inhibitors and cancer therapy. Recent Results Cancer Res. 172, 25-44 (2007).

70. Kariyawasam, H.H. et al. Activin and transforming growth factor-beta signaling pathways are activated after allergen challenge in mild asthma. J. Allergy Clin. Immunol. 124, 454-462 (2009).

71. Trapnell, C. et al. Differential gene and transcript expression analysis of RNA-seq experiments with TopHat and Cufflinks. Nat. Protoc. 7, 562-578 (2012).

72. Meyer, L.R. et al. The UCSC Genome Browser database: extensions and updates 2013. Nucleic Acids Res. 41 (Database issue), D64-D69 (2013).

73. Robinson, D.R., Wu, Y.M. \& Lin, S.F. The protein tyrosine kinase family of the human genome. Oncogene 19, 5548-5557 (2000). 